\title{
Mining information from atom probe data
}

\author{
Julie M. Cairney a,b,*, Krishna Rajan ${ }^{\mathrm{c}}$, Daniel Haley ${ }^{\mathrm{d}, \mathrm{e}}$, Baptiste Gault ${ }^{\mathrm{d}}$, Paul A.J. Bagot ${ }^{\mathrm{d}}$, \\ Pyuck-Pa Choi ${ }^{\mathrm{e}}$, Peter J. Felfer ${ }^{\mathrm{a}, \mathrm{b}}$, Simon P. Ringer ${ }^{\mathrm{a}, \mathrm{b}}$, Ross K.W. Marceau ${ }^{\mathrm{f}}$, \\ Michael P. Moody ${ }^{\mathrm{d}}$
}

\author{
a School of Aerospace, Mechanical, Mechatronic Engineering, The University of Sydney, NSW 2006, Australia \\ ${ }^{\mathrm{b}}$ Australian Centre for Microscopy and Microanalysis, The University of Sydney, NSW 2006, Australia \\ ' Department of Materials Science and Engineering, Iowa State University, Ames, IA 50011, USA \\ d Department of Materials, University of Oxford, Parks Road, Oxford OX1 3PH, UK \\ e Max Planck Institut für Eisenforschung GmbH, Max-Planck Straße 1, 40237 Düsseldorf, Germany \\ ${ }^{\mathrm{f}}$ Institute for Frontier Materials, Deakin University, Geelong Technology Precinct, 75 Pigdons Road, Waurn Ponds, Victoria 3216, Australia
}

\section{A R T I C L E I N F O}

\section{Article history:}

Received 13 November 2014

Received in revised form

3 May 2015

Accepted 12 May 2015

Keywords:

Atom probe tomography

Microscopy

Data mining

Clustering

Short range order

Crystallography

\begin{abstract}
A B S T R A C T
Whilst atom probe tomography (APT) is a powerful technique with the capacity to gather information containing hundreds of millions of atoms from a single specimen, the ability to effectively use this information creates significant challenges. The main technological bottleneck lies in handling the extremely large amounts of data on spatial-chemical correlations, as well as developing new quantitative computational foundations for image reconstruction that target critical and transformative problems in materials science. The power to explore materials at the atomic scale with the extraordinary level of sensitivity of detection offered by atom probe tomography has not been not fully harnessed due to the challenges of dealing with missing, sparse and often noisy data. Hence there is a profound need to couple the analytical tools to deal with the data challenges with the experimental issues associated with this instrument. In this paper we provide a summary of some key issues associated with the challenges, and solutions to extract or "mine" fundamental materials science information from that data.
\end{abstract}

c) 2015 Published by Elsevier B.V.

\section{Introduction}

Atom probe is a powerful characterisation tool that provides three-dimensional reconstructed maps that show the position and species of tens or even hundreds of millions of individual atoms with atomic resolution [1-6]. Such datasets are rich in information that is of great use to materials scientists, who are interested in the relationship between structure and properties of materials, but it is a major challenge to convert this data to quantitative and meaningful information about the microstructure of the specimen being examined.

A needle-shaped specimen is biased at a high voltage and the resulting electrostatic field causes ionisation and desorption of the surface atoms. By partially pulsing the field, a few ions at a time are projected onto a position-sensitive, single atom detector. The position at which they hit the detector is used to reconstruct the

\footnotetext{
* Corresponding author at: School of Aerospace, Mechanical, Mechatronic Engineering, The University of Sydney, NSW 2006, Australia.

E-mail address: julie.cairney@sydney.edu.au (J.M. Cairney).
}

$x-y$ coordinates, and the sequence in which the ions are detected is used to reconstruct the $z$ coordinate. The time-of-flight is used to identify the atomic species. A typical dataset, once reconstructed, is in the form of a point cloud containing the 3D position and mass-to-charge state of each ion. Visualisation may be carried out by using either dedicated commercial software, such as IVAS (from CAMECA), or by other software (e.g. Blender or 3Depict) that has the capability of rendering the point cloud data as points or spheres and colouring the atoms according to the mass-to-charge ratio, and therefore the identified species. Many of these software packages already provide sophisticated tools for the quantitative analysis of data, such as grid-based atomic distributions [7] and rendering of iso-surfaces, concentration profiles, proximity histograms [8], cluster analysis tools [1-9] and the analysis of multiple hit detector events [11-13]. The purpose of this paper is to outline some outstanding issues and describe some of the more recent developments for mining information from atom probe data, based on a "data mining" workshop held at the Atom Probe Tomography and Microscopy (APT\&M) meeting in Stuttgart, August, 2014. 


\section{Defining and assessing randomness}

A true nanoscale characterisation technique, atom probe enables clusters of solute/dopants/impurities consisting of mere handfuls of atoms to be identified and characterised [14]. Of course, the natural inclination is to push this capacity to its limits. A prominent example is the investigation of nucleation during the early stages of phase transformations in materials [15-19]. Other popular applications include the onset of segregation of solute ions to defects in materials subject to extreme environments, such as ion/neutron irradiation [20-23], and the clustering of solute ions in semiconductor devices [24-27].

At these limits of APT sensitivity and resolution, researchers are examining the distribution of very fine-scale clusters of atoms and effectively asking - is this distribution of atoms random? More precisely, is it probable that this distribution could be observed in a completely randomly arranged configuration of atoms? Although this might seem like a straightforward proposition, given the intrinsic limitations of experimental data, this interpretation requires careful consideration.

Visual inspection of the reconstruction is nearly always the first course of action. In many systems chemical-spatial inhomogeneity is readily apparent. However, discriminating very slight deviations from random by eye is simply impossible. Iso-concentration surfaces provide significant enhancement to visual inspection. Isoconcentration surfaces can identify regions of irregular chemical composition that may differ only very slightly from the bulk of the reconstruction. However, this information alone is not sufficient to determine whether these regions are statistically significant, i.e. are they evidence of the first stages of the nucleation of an entirely new phase, or simply a random fluctuation?
For example, a computer-generated face centred cubic solidsolution model system has been generated, and is shown in Fig. 1. The simulated system consists of two types of solutes, solute A (8.0 at\%) and solute B (1.5 at\%). Both types of solute atoms have been randomly distributed throughout the matrix. Even so, application of an iso-concentration surface to this system highlights discrete regions of high solute composition. Indeed the spatial distribution of these regions around the reconstruction appears very much like what might be expected for a population of secondary phase clusters homogeneously nucleating within the material. Further, secondary analyses such as proxigrams can easily be applied that ostensibly strengthen the notion that this is evidence for fine scale thermodynamically-driven solute clustering.

However, in this example we already know that this is not true. The fluctuations are simply random variations in the composition. The key point is that without some kind of statistical analysis to support the hypothesis that clustering is present, the iso-concentration surface is meaningless. Conventionally, frequency distribution analyses, often colloquially referred to as binomial analyses, have been applied as a first test for randomness [28,29]. Frequency distribution analyses essentially divide the APT data into a 3D grid of voxels of similar size, all containing the same number of atoms. The frequency of occurrence of a specific element per voxel is assessed. Ultimately a histogram of these frequencies is constructed. If an element is randomly distributed throughout the reconstruction, the histogram should closely match a corresponding theoretical binomial distribution. Hence, non-randomness can be assessed by quantifying the departure of the experimentally observed distribution from that expected theoretically. Often a $\chi^{2}$ test is applied to obtain a $p$-value and test the significance of this deviation. However, the sensitivity of this

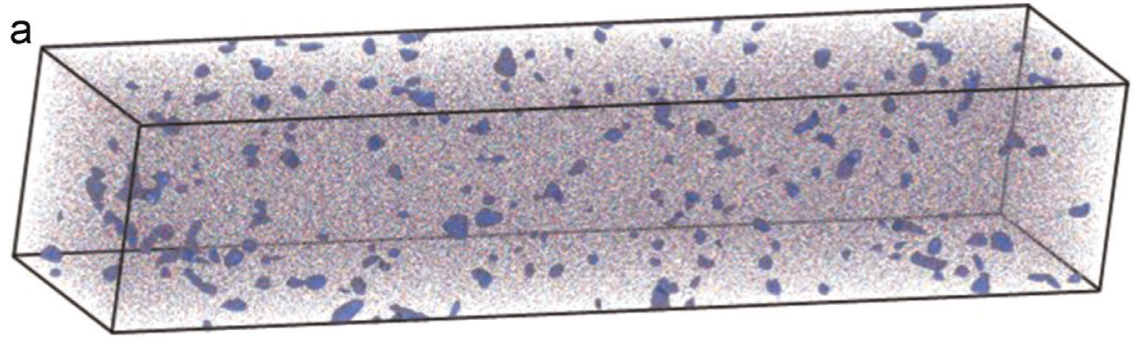

b

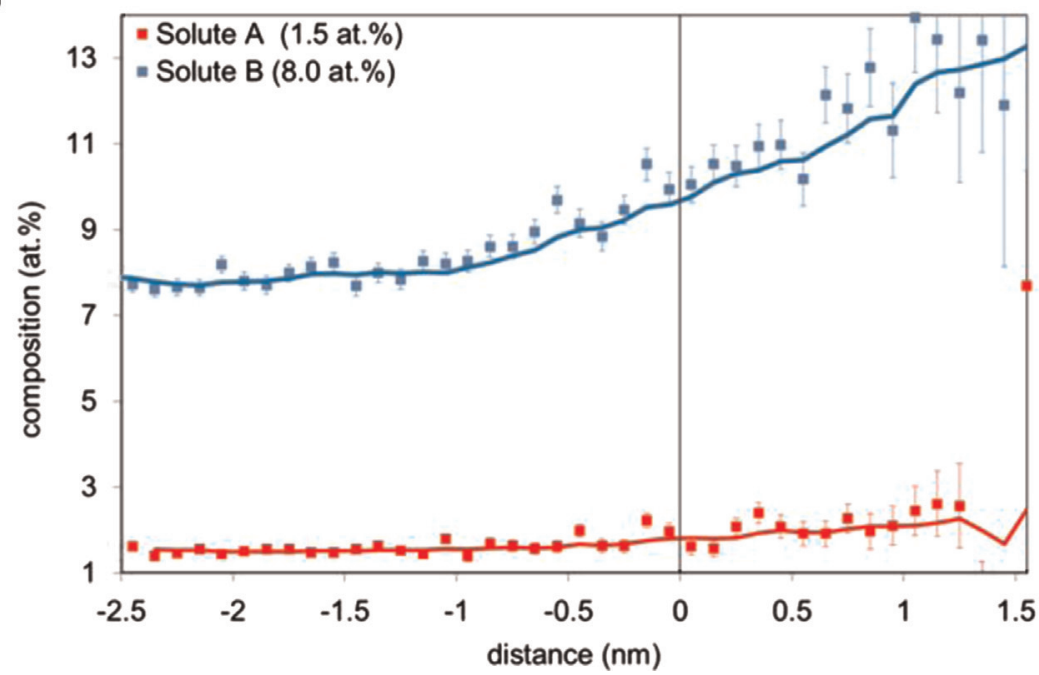

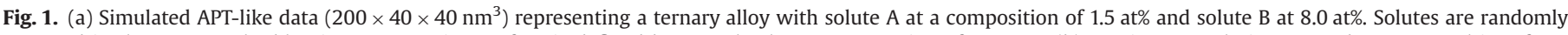

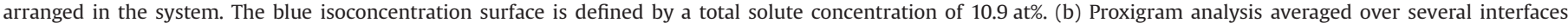
defined by 10.9 at\% isoconcentration. (For interpretation of the references to colour in this figure legend, the reader is referred to the web version of this article.) 
a

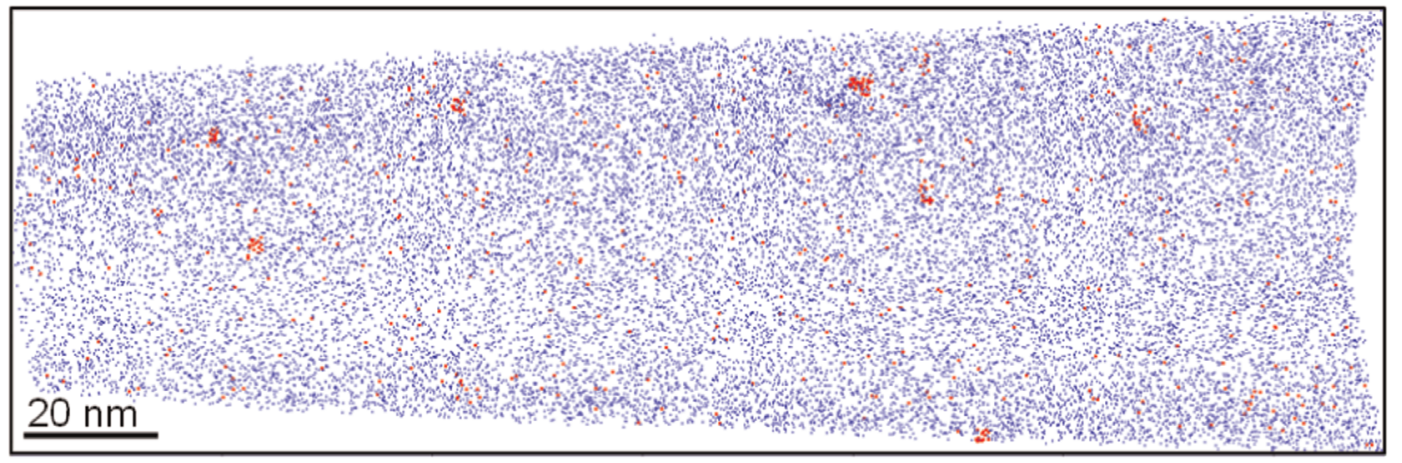

b

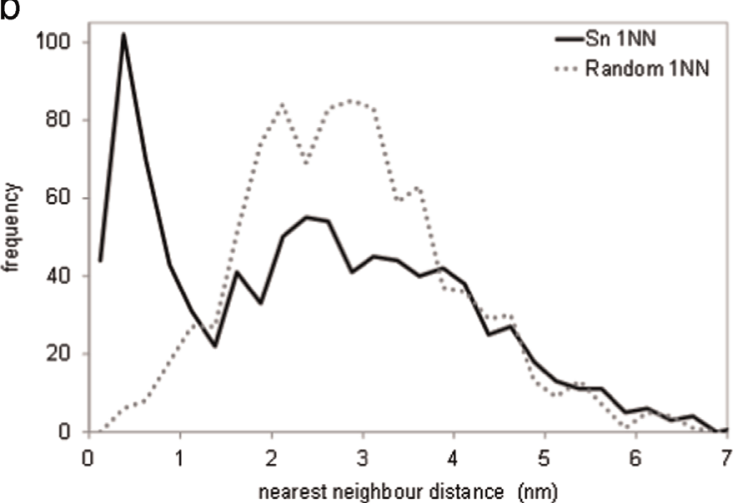

C

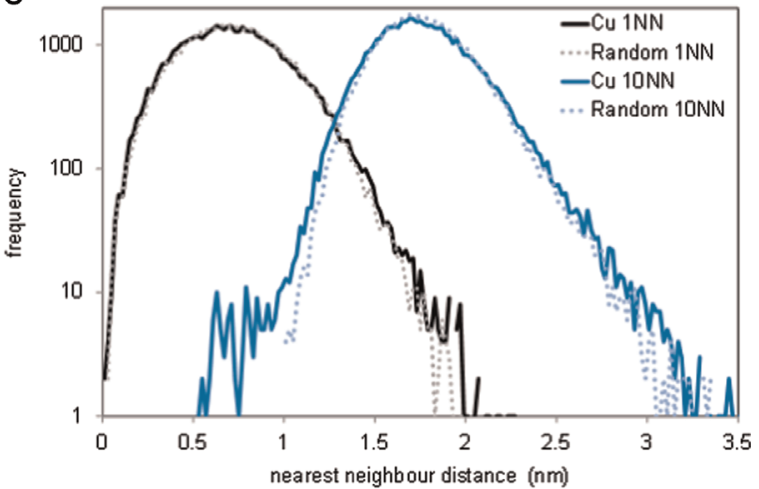

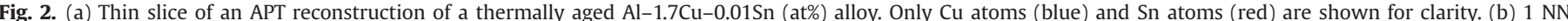

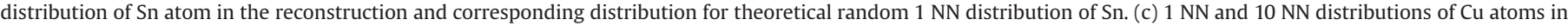

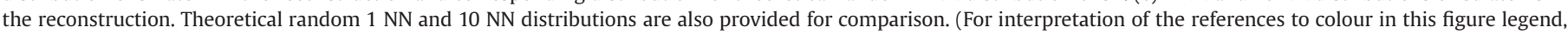
the reader is referred to the web version of this article.)

approach can depend on the size of the voxels in to which the data is divided at the start of the analysis [30]. The optimal size of the voxel is ultimately a balance between the size of the dataset, the concentration of the element of interest, and the scale of clustering in the reconstruction. A voxel size that is too large will blur positional information and may obscure very fine-scale clustering from the analysis. Conversely voxels that are too small may introduce statistical error with respect to composition. It is also known that for large data sets that, as their size continues to increase, so too does the likelihood that the $\chi^{2}$ test will erroneously indicate a nonrandom distribution [30]. Since the value of $\chi^{2}$ depends on the size of the dataset, Pearson's coefficient, $\mu$, should be adopted to normalise this value and to make direct comparisons between the amount of clustering in different sized reconstructions [31]. However, it is not possible to infer randomness directly from the value of $\mu$ alone.

Examining the distribution of nearest neighbour (NN) interatomic distances is a simple concept, but one which has proven effective for investigating solute clustering phenomena [32]. The NN distribution of a particular solute within a reconstruction can be compared to the distribution obtained from a complementary randomised data set. Deviation of an experimentally-obtained NN distribution from the comparator indicates a non-random distribution of solute, for example the Sn distribution in Fig. 2.

The NN distribution can be considered to incorporate distributions from two distinct sets of distances: the NN distances between clustered solute atoms and the NN distances. Analytical methods have been developed to extract these two component distributions from the experimental distribution and quantify the contribution from each $[33,34]$. However, in the case of detecting the onset of very fine-scale clustering no standard means has been established to quantify small deviations between the experimental and randomised distribution and then to subsequently apply some threshold to define the onset of significant clustering.

When examining the distribution of a particular element, the first nearest neighbour will become increasingly less sensitive to slight departures from randomness with increasing concentration. This is simply because it is increasingly likely that a solute will have a close near neighbour regardless of whether it sits in the matrix or is incorporated into a cluster. An example of this is the comparison of the $\mathrm{Cu}$ and $\mathrm{Sn}$ distributions in Fig. 2b. Analysis of higher order nearest neighbour $(k N N)$, e.g. 10th nearest neighbour (10 NN), can provide effective insights in this case [34]. The choice of which order, $k$, of nearest neighbours to investigate is analogous to selecting a voxel size for the frequency distribution analysis. Optimisation of the NN order will depend on the concentration of the element of interest, using higher order kNN may reduce statistical fluctuations but at the expense of increasingly losing local information describing the neighbourhood surrounding each atom.

The natural extension to $k \mathrm{NN}$ analyses are cluster identification algorithms, which define and characterise the nature of discrete nanostructural features [35]. The application of clustering algorithms underpins a significant number of atom probe analyses. As such there is an increasing amount of literature on the different algorithms that have been developed and how these might be best parameterised for specific types of application. Marquis and Hyde [9] and Gault et al. [1] provide overviews. Hence it is not discussed in detail here, suffice to say that application of these techniques to determine the very onset of clustering all parameters must be chosen with extreme care to optimise sensitivity. Furthermore, as is the case for isoconcentration surfaces, without appropriate statistical analysis and some kind of threshold metric (e.g. a random comparator system), the identification of clusters is not proof 
on its own of significant non-randomness.

Finally, it is important to acknowledge limitations of the technique when undertaking this kind of investigation. For example, certain elements are prone to surface migration during the experiment $[36,37]$. The most prominent example of this is solute segregation to crystallographic poles. In some circumstances, these effects can be mitigated by adjusting the experimental setup. At the very least regions exhibiting the effects of surface diffusion must be removed from the data prior to statistical analysis. The potential effect of limited detection efficiency is often overlooked. It has been shown that the frequency distribution is susceptible to this influence, with the analysis indicating an increasingly randomised distribution with decreasing detection efficiency [1]. Similarly $k N N$ distance and cluster-size distributions would be expected to increasingly tend towards a random distribution.

\section{Short-range order}

Short-range order (SRO) is typically characterised in terms of well-established SRO parameter formalism [38,39] employing probability and concentration terms from counting statistics, and is commonly measured experimentally using bulk, volume-averaged techniques (e.g. X-ray scattering, neutron scattering and Mössbauer spectroscopy) to determine the apparent structure. APT data, on the other hand, provides a unique combination of highly resolved atomistic information (compositionally and spatially) in three dimensions (3D), which can be directly data-mined to extract quantitative structural information in the context of a generalised multi-component short-range order (GM-SRO) parameter [40]. GM-SRO analysis allows higher-order atom correlations (pairwise and upwards) in simple binary and multicomponent systems, and is carried out on experimental APT data by shell-based counting of the atoms at discrete 3D radial distances, accounting for limited detector efficiency and spatial resolution from the instrument [41]. In fact, this atom-by-atom analysis capacity has a very similar construction to that of a radial distribution function (RDF) for APT data [42-45], including chemical species-specific "partial RDFs" and pair correlation functions, all of which extend the application of 3D atom probe data from statistical analysis using 1D Markov chains and the Johnson and Klotz ordering parameter [29,46-49] to a wealthier description of SRO that compliments existing capability for long-range order (LRO) and site occupancy investigation [26,50-59].

By standard convention, a positive value of a GM-SRO parameter defines co-segregation for a particular set of elements in a certain crystallographic shell, which indicates clustering, whereas a negative value indicates anti-segregation (ordering) of the two sets of elements $[40,41]$. According to the formalism, a random configuration nominally occurs when this parameter equals zero. Whilst the definition of LRO is quite clear and refers to repetition of crystal lattice periodicity, SRO is less clear with respect to the exact extent of the atomic order. Interestingly, however, there exists a definition for medium-range order (MRO) as used within the amorphous materials community, which refers to order that extends over intermediate distances, beyond the second or third atomic shell, e.g. $1-2 \mathrm{~nm}[60,61]$.

Recent work by Marceau et al. [62], presented at APT\&M in the data-mining workshop, demonstrated GM-SRO analysis of both simulated and experimental APT data in the classic orderingtransformation $\mathrm{Fe}-\mathrm{Al}$ system. Here, GM-SRO parameters from the 1 st to 5th coordination shells were determined for this simple binary case (lattice parameter bcc-Fe $=2.863 \AA$ ) having $\mathrm{Fe}-\mathrm{Al}, \mathrm{Al}-$ $\mathrm{Fe}, \mathrm{Fe}-\mathrm{Fe}$ and $\mathrm{Al}-\mathrm{Al}$ interactions. In order to firstly validate the approach, the method was tested on a simulated dataset of 128,000 atoms and composition $75 \% \mathrm{Fe}$ and $25 \% \mathrm{Al}$, having the $\mathrm{D}_{3}$ crystal lattice with known pairwise SRO parameters. Three cases were investigated and compared: (i) the perfect $\mathrm{DO}_{3}$ crystal lattice; (ii) the effect of limited detector efficiency by random removal of $70 \%$ of the atoms; and (iii) the combined effects of $30 \%$ detector efficiency and spatial noise to simulate real experimental APT data. Spatial noise was applied with a $0.1 \mathrm{~nm}$ FWHM spherical Gaussian probability distribution to randomly displace each atom from the perfect $\mathrm{DO}_{3}$ crystal lattice. It was revealed that even after random removal of $70 \%$ of the atoms, the various GM-SRO parameters were re-captured very well from the 1 st to 5 th shells. However, when effect of spatial noise was superimposed on top of that of $30 \%$ detector efficiency, despite the sign of the GM-SRO parameters remaining consistent in all cases (i.e. positive for co-segregation/ clustering or negative for anti-segregation/ordering), only the $1 \mathrm{st}$ shell information was accurate, and all other GM-SRO values became very small (close to zero, i.e. random occupancy), compromising the integrity of the analysis. Simply stated, the combination of imperfect spatial resolution with limited detector efficiency has the effect of making the APT data appear more randomised. It is important to remember however, that few (if any) other experimental measurement techniques can, with the aid of data mining, produce chemical species-specific SRO analysis of atomic-scale 3D data.

In order to improve the accuracy of the GM-SRO analysis extending to higher order coordination shells, lattice rectification of the reconstructed atom probe data was carried out prior to application of the GM-SRO analysis. Lattice rectification uses crystallographic information from the APT data reconstruction to restore the lattice-specific atomic configuration of the original specimen $[63,10,142]$. Again, to assess the feasibility of this method, it was applied to the simulated $\mathrm{DO}_{3}$ crystal data, having "atom probe-like" spatial noise and detector efficiency. Initial findings on the simulated data indicate that lattice rectification improves the GM-SRO measurements and can provide an estimation of the spatial noise tolerance [62].

\section{Atom probe crystallography}

It is well known that atom probe data collected from crystalline material often contains information from the original crystal lattice. In the early days of atom probe, crystallographic information was obtained through a comparison with the terraces of atoms visible in field ion microscopy (FIM) images [64,65]. However, there is also crystallographic information contained within atom probe data itself. This information takes two main forms. Firstly, the dataset as a whole contains density fluctuations that can be the result of trajectory aberrations due to variations in the electric field at the edges of atomic terraces. An extreme example for a dataset from pure aluminium is shown in Fig. 3a. Images like this are often referred to as 'field desorption images' and can be obtained by either displaying a detector hit-map, or showing a slice of data taken perpendicular to the analysis direction (along the $z$ axis) from a reconstructed dataset. Strictly speaking, the latter is not a "field desorption image", and will have a different scale, but the term is often used to describe either of these two types of images. The image in Fig. 3a is a slice through the data, and is coloured according to the density of the ions (a 2D density map).

The second way in which information about the lattice is manifest in atom probe data is in the form of planes, which can be visualised when the dataset is oriented with the planes parallel to the viewing direction. These planes are generally observed in the vicinity of crystallographic "poles" in the field desorption maps, i.e. regions in which a plane is parallel to tangent of the curved surface of the sample. They are not usually visible across the entire specimen. The images to the right of the 2D density map in Fig. 3a 
a

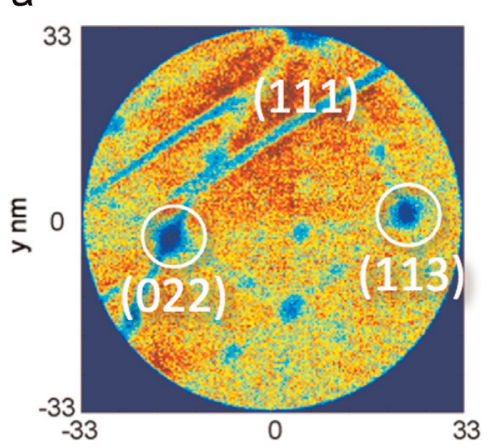

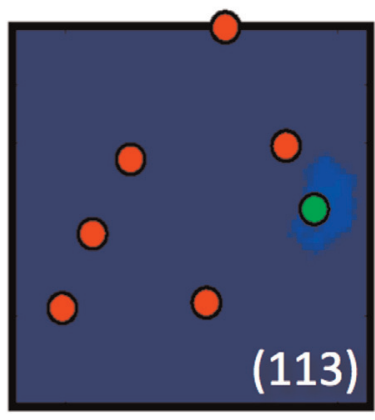

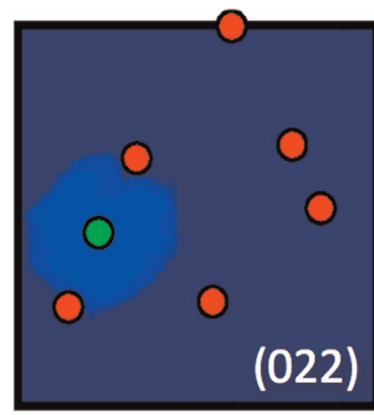

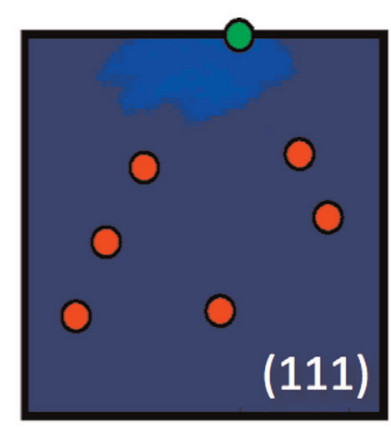

b

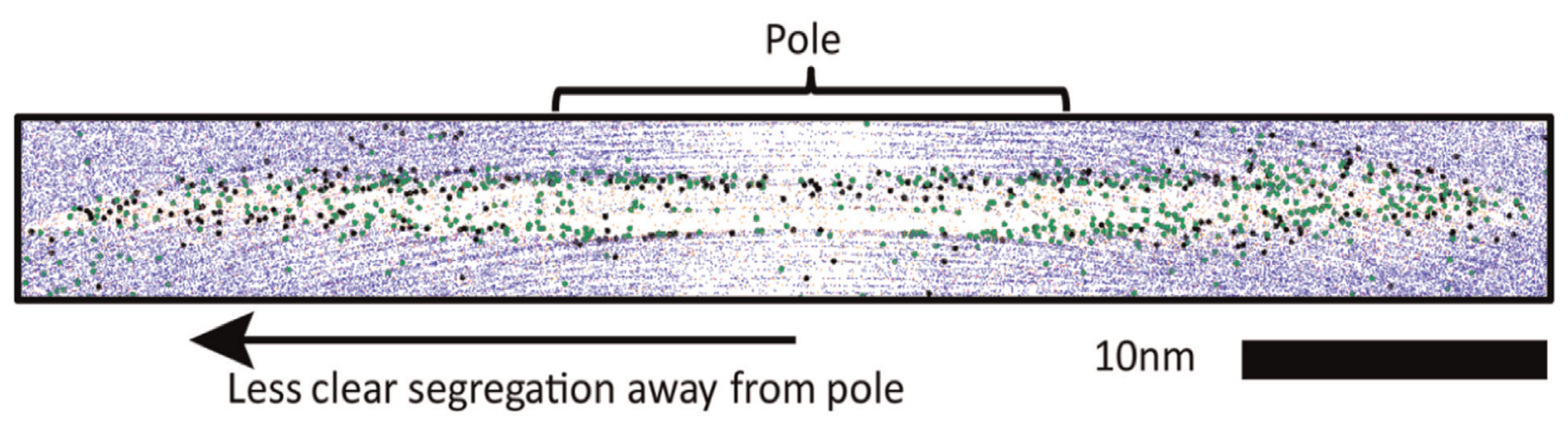

C

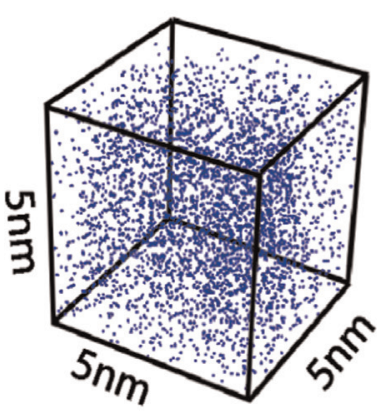

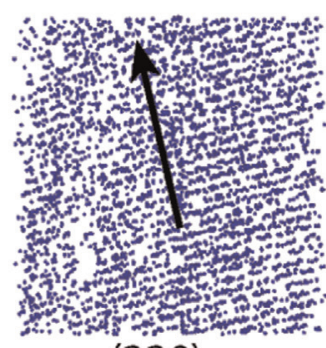

(220)

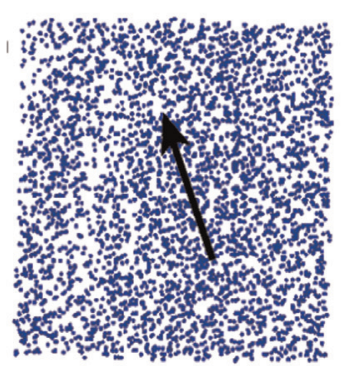

(331)

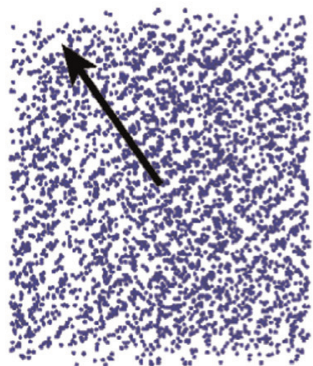

(111)

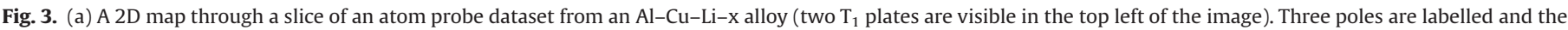

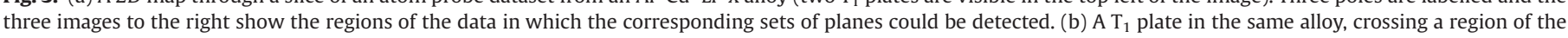

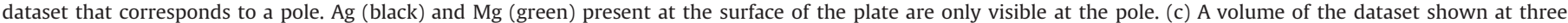

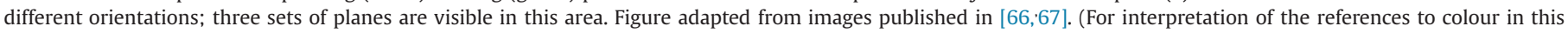
figure legend, the reader is referred to the web version of this article.)

show the regions of the data in which the corresponding sets of planes can be detected (the figure was generated using methods described in [67]).

For this reason, the resolution in the analysis directions ( $z$ direction) is different across an atom probe dataset, being highest in the vicinity of the poles [68]. This is shown in Fig. 3b, which shows a $\mathrm{T}_{1}$ platelet in an $\mathrm{Al}-\mathrm{Li}-\mathrm{Cu}$ alloy that is positioned across a region of the dataset that corresponds to a pole [66]. Ag (black) and $\mathrm{Mg}$ (green) atoms are present at the surface of the plate, but this is only visible in the centre of the pole. Away from the pole the resolution is insufficient to show the planes or the surface layers. Various approaches have been proposed to detect the planes in reconstructed 3D datasets, including via Fourier transforms [6971], Hough transforms [72], or spatial distribution maps (SDMs) $[59,68,73]$. Interestingly, it is occasionally possible to observe more than one set of planes in the same area. This is evidenced in the production of 2D spatial distribution maps [59,68,73]. Further evidence is provided in Fig. 3c, which shows a $5 \mathrm{~nm} \times 5 \mathrm{~nm}$ volume in an Al-alloy that contains 3 distinct sets of planes [67].

This crystallographic information is useful in a number of ways. Methods proposed by Gault and co-workers [74,75] to use the field desorption image to calibrate the compression factor and the field factor by using geometrical projections from the desorption images has now become standard practise for the calibration of atom probe datasets, when this information is present. Information about the location of the different poles at the tip has the potential to form the basis for a global reconstruction tool that may account for uneven shifts in the position of atoms in the $\mathrm{x}$ and $\mathrm{y}$ directions across the sample, due to the non-hemispherical nature of real atom probe tips [67]. Crystallographic information from the atom probe data can also be used to provide the basis for lattice rectification, where atoms are shifted to their most likely original position [63].

If several planes can be identified, it is possible to determine the overall lattice orientation of the grains within the sample investigated $[76,77]$. This information can be extracted directly from field desorption images $[1,74,78]$, or from reconstructed 3D datasets via the approaches described above [59,68-73]. If several grains are present, it is possible to provide information about the "nanotexture", or the relative orientation of the grains, including complete details of the grain boundary orientation relationships $[77,79]$. It is possible to colour images according to an inverse pole 
figure to provide orientation maps. Knowing the orientation of grains also provide information about the crystallography of microstructural features such as dislocations $[80,81]$, precipitates, or grain boundaries.

Not all samples yield data that contains crystallographic information. While pure metals or ordered alloys usually display density fluctuations and planes (the two normally go hand in hand), heavily alloyed materials and non-conductors tend to provide data that contain little, or are devoid of, crystallographic information. There are exceptions to these rules [26,82], and clear criteria for which specimens might retain lattice information have not been established. Whilst many approaches to extracting crystallographic information have been proposed, most require manual selection of small volumes in the vicinity of poles. Different areas are separately interrogated until sufficient information is collected. This is time consuming, and does not provide a definitive answer as to whether a given dataset contains sufficient crystallographic information to determine the crystal orientation. At APT\&M 2014, Araullo-Peters et al., presented an automated approach that utilises SDMs to interrogate all regions of the sample, and extract any crystallographic data that is present [67]. For selected points throughout the dataset, an SDM is computed, identifying the orientation and interplanar spacings for any detectable planes. It outputs a complete list of the planes that are detected, and their orientation within the dataset (as well as which region of the dataset they were observed). This program can be used to (a) assess whether sufficient information is present and (b) capture the full extent of crystallographic information contained within the dataset for further interpretation.

\section{Computational geometry approaches to delineating micro- structural features}

Many of the features in atom probe data, such as grain and phase boundaries [83-89], thin layers [90-94] and dislocations decorated with solutes are readily visible in 3D atom maps. However, quantitative analysis of the distribution of the atoms with respect to these features is required for a meaningful interpretation of the data [8,95-97]. While the definition of such objects is an abstraction [98], it is required for improved visualisation or to relate the distribution of the atoms to these features. This demands the derivation of a geometric model for the feature, or even a network of features, represented by a piecewise linear approximation $[8,99,100]$.

A piecewise linear approximation is the combination of a set of points that delineate the feature(s) and some representation of how these points are connected. The connectivity is usually represented in a list of indices of these points, with each list entry defining a triangle (surfaces or interfaces) or line element (e.g. dislocation), or as a connectivity matrix. This is referred to as the "mesh topology". The representation as points ("vertices") and triangles/lines ("faces"/“edges"), is widespread in the computer graphics community and used in popular file formats to exchange 3D data (e.g. Wavefront *.obj, Stanford *.stl and *.ply). The use of triangle lists also allows for the definition of the orientation of the surface ("inside" and "outside"), by implicitly defining the surface normal by the order of the indices (right hand rule).

Based on this delineation of a feature, local coordinate systems can be derived, in order to relate the location of the atoms within the dataset either to their distance relative to the feature or the location along the feature. The former, referred to as the proximity histogram in atom probe tomography [8], is one of the most established and widely used techniques to in APT while the latter, in the form of interfacial excess and concentration maps is a more recent development [101-104].

\subsection{Feature extraction (iso-surfaces and DCOM)}

In order to obtain a model of an interface or surface, different approaches have to be chosen depending on whether the feature is characterized by a local gradient of some property or a local maximum or minimum. Often, atomic concentration or density of a species serve as these properties, but also the density variations caused by reconstruction artefacts and the like can be used $[105,106]$.

In the case where the feature is characterized by a local gradient, iso-surfaces can be used, which are produced by the marching cubes algorithm [99]. This algorithm extracts an isosurface from a voxelisation of the data, a 3D histogram in which the data is divided in small rectilinear volumes. The iso-surface is defined by choosing a threshold value, above which the voxel is considered to be "inside" the surface and creating a surface around these voxels. Since it is known how these voxels are connected, a simple scheme can be used to place the surface points and create the triangles based on a look-up table.

For features which are characterized by a local maximum in concentration (e.g. grain boundaries or very thin films), manual analysis approaches are often used to measure the concentration, such as cropping out volumes of data and measuring concentration profiles [82]. However, the result depends on the choice of analysis location and fails in the case of highly curved surfaces. A two-step approach has been developed to define such objects. First, an approximation of the feature is derived by either manually placing points by using computer graphics software (e.g. Blender), or using semi-automated mesh generation algorithms [107]. Secondly, the surface is better fitted to the feature by minimisation of the distance between the centre of mass (DCOM) of a selected species of atoms within a spherical volume and the centre of the volume [104]. The process is described in Fig. 4. This is effective for the delineation of features that are narrow in at least one direction, such as grain boundaries, dislocations or gate oxides in transistors. a
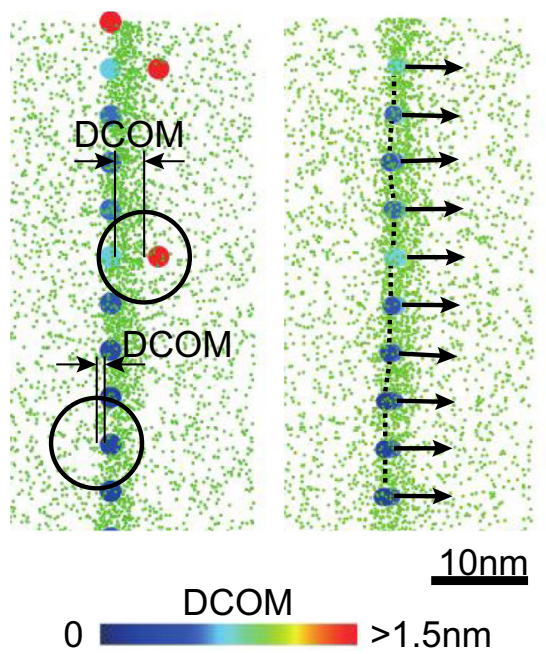

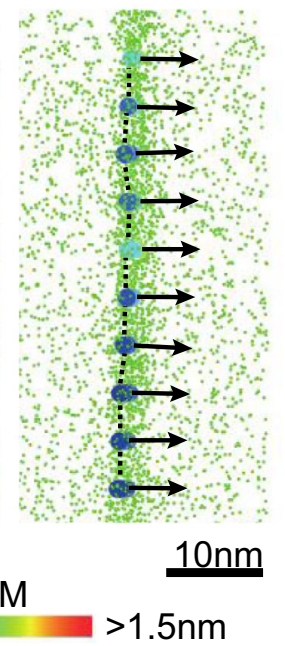

b

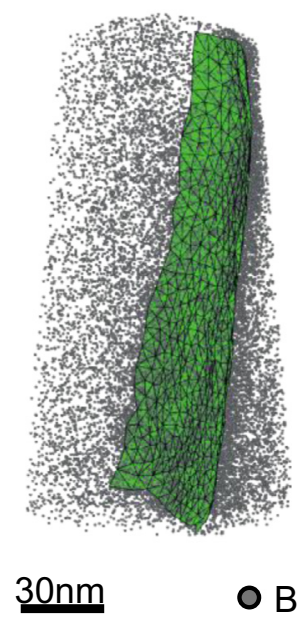

Fig. 4. DCOM: (a) the principle of the DCOM method to extract features with segregation. In order to move the vertices of an analysis mesh onto a feature, the distance between the centre of a sphere and the centre of mass of the contained atoms of interest is iteratively minimised. (reproduced from [104] with permission from Elsevier). (b) A grain boundary in a super duplex steel outlined using the DCOM approach. 
a
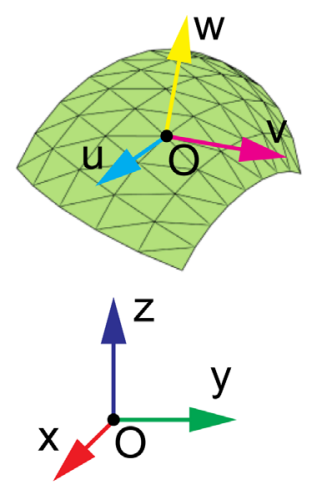

b

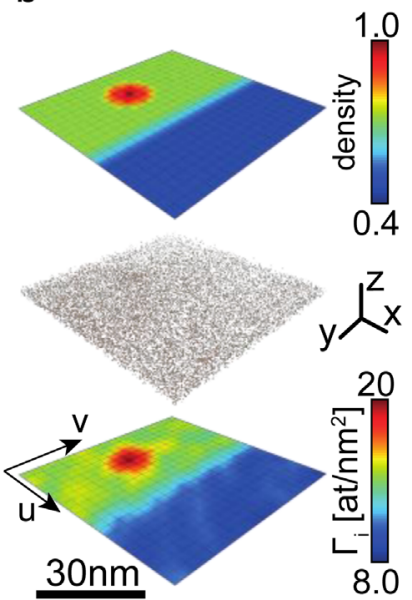

C
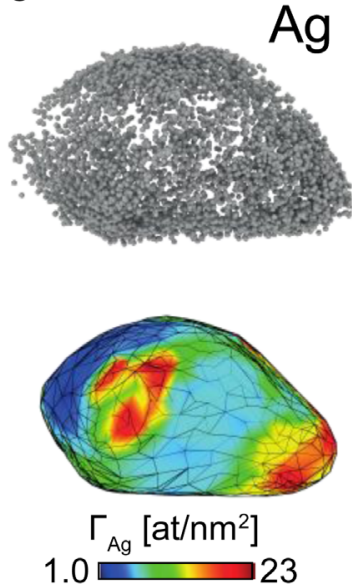

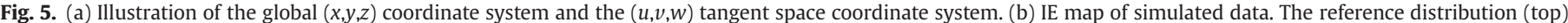

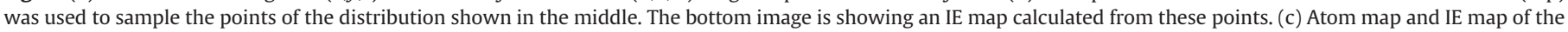
Ag distribution on the surface of a Au@Ag nanoparticle (with permission from Wiley [103]).

\subsection{Proximity histograms and interfacial excess mapping}

The surfaces derived with the algorithms described above can then be used to provide quantitative data. The surfaces act as reference coordinate systems $(u, v, w)$ for the atoms within the APT dataset, allowing for the calculation of the distance to the surface ( $w$ coordinate) as well as the projected lateral location of each atom ( $u, v$ coordinates) on the surface (Fig. 5a). The lateral location of an atom on the surface is defined as the location of the closest surface point, since the atomic positions do not precisely coincide with the surface. Similar coordinate systems can be defined for line-like objects (e.g. dislocations or grain boundary junctions) and point-like objects (e.g. clusters) [104]. In practise, however, the $u, v$ coordinates are discretized, meaning local properties are calculated for each mesh vertex. Knowing the distance of each atom to a surface ( $w$ coordinate), a histogram of atomic concentration or other properties with respect to the surface can be calculated $[8,97]$.

Often the lateral distribution of a species on a surface or within a thin film is of interest [108]. This is referred to as an interfacial excess (IE) map. An IE map quantifies the lateral distribution of a segregated species by summing the number of atoms per unit area over the thickness of the segregation zone and subtracting the bulk concentration of these atoms $[83,98,109,110]$. The need for this type of analysis arises from the fact that even qualitative judgment about whether a significant variation in segregation or thickness is present cannot be based on visual inspection alone. This is illustrated in Fig. 5b where, based on the reference density distribution on the top, a point distribution of 50,000 points was generated. Even though this distribution has a $60 \%$ variation in it, no variation is visually apparent in the rendering of the point distribution. The IE map however, matches the reference distribution reasonably well.

The calculation of IE maps demands the mapping of species in a non-flat $2 \mathrm{D}$ coordinate systems, generally referred to as $u, v$ coordinates. This type of analysis was first facilitated by Felfer et al. $[101,104]$ by calculating a Voronoi decomposition of the dataset [111], with the vertices of the analysis mesh as the generating points of the decomposition. Using this approach, quantitative maps of the distribution of species in thin film layers [104], grain boundaries [101,102,112] and on nanoparticle surfaces [103] have been calculated. By using a Voronoi decomposition, quantitative values can be derived even for highly curved surfaces, but care needs to be taken regarding the distribution of the mesh vertices in order to avoid small analysis volumes around individual vertices.

As an example, a map of Ag on the surface of an Au@Ag coreshell nanoparticle is shown in Fig. 5c [103]. Due to the highly curved nature of the particle surface, the variations in Ag coverage are hard to discern in the Ag distribution, whereas the Ag IE map clearly shows the locations of higher and lower coverage.

Yao et al. [102,112] used a similar approach to map IE by placing aligned boxes at the location of each mesh vertex. The advantage of the aligned box approach used by Yao et al. is that the determined local values are largely independent of the distribution of the vertices on the surface. Drawbacks are that it can easily result in artefacts when analysing highly curved surfaces (self intersection) and it cannot be used to quantify the overall amount of segregation due to overlaps of the boxes.

\section{Spectral decomposition in atom probe}

Atom probe is a powerful mass-spectroscopy technique. The mass-to-charge ratio associated with each event is accumulated into a histogram, providing a spectrum of the identities within the dataset. Users working with atom probe data utilise this spectrographic data in order to construct a mapping between specific mass ranges within the spectrum to a given atomic identity, a process known as "ranging".

The mapping at this point is sufficient if an unambiguous label can be assigned to a given mass range. However, this is sometimes not possible. Different species, such as ${ }^{58} \mathrm{Ni}$ and ${ }^{58} \mathrm{Fe}$ in either the $1^{+}$or $2^{+}$charge states, can have sufficiently similar masses to make unique identification from the spectra impossible with current systems.

Indeed, to discriminate between these two in a current atom probe design a very high mass resolution would be required. $\mathrm{Ni}$ has a mass of 57.9353 and $\mathrm{Fe}$ a mass of 57.9332 , thus requiring resolution of $<0.002 \mathrm{Da} /$ charge $(1+$ state) or $<0.001 \mathrm{Da} /$ charge $(2+$ state $)$ to fully resolve the two. This difference is far below the timing resolution, and thus mass resolution, of atom probe experiments, where reachable resolutions are on the order of $0.05 \mathrm{Da} /$ charge, depending upon position in the spectrum. Furthermore, these overlaps can occur due to the overlap of differing charge states, and are thus not simply restricted to similarly 
massed isotopes, such as in the case of ${ }^{16} \mathrm{O}^{+}$and ${ }^{32} \mathrm{~S}^{2+}$.

The timing (and thus mass) resolution of atom probes is limited by many sources of error, such as the velocity spread of ions leaving the tip, random deviations between the initial pulse and the evaporation time of an ion, as well as limited detector timing resolution. Some of these can be mitigated, such as via time focussing in reflectron equipped systems in order to compensate for velocity spread $[113,114]$ and by flight path extension for detector timing limited systems. Other physical phenomena, such as pulse-evaporation deviations cannot be so readily accounted for, and may fundamentally limit the achievable resolution. Thus identification of species from APT can remain ambiguous in certain cases.

This issue has generated proposals to largely circumvent this resolution problem, such as the use of alternate information that could hypothetically be acquired with new detector designs, such as kinetic energy detectors [115]. However this is not yet a proven technology in atom probe. Data analysis based approaches, such as the so-called multiple event discrimination [11], and multivariate analysis [116] are aimed at using secondary data to split the overall spectra into several sub spectra, where these sub-spectra may not be affected by overlaps. More typically, users may partition datasets into several sub volumes manually, such as if several phases are present within a dataset, and then perform ranging on each spectra to interpret these results.

These methods are only viable in the case that some kind of secondary information is available to act as a "key" to break the spectra into parts (e.g. spatial or multiple hit data), so that contributions can be unambiguously identified. Indeed, several APT works cite the inability to resolve overlaps as a concern in their analyses [117-119].

However, this limitation does not prohibit the computation of the overall composition of datasets, due to a technique known as "decomposition" or "deconvolution" [4,29], which uses basis peak components derived from natural isotope abundances, then solves them for composition. Such calculations can be expressed using simple matrix models [1], or more recently "blind" methods taking into account peak shape have been discussed [120]. However standard decomposition techniques, such as implemented in APT software programs solve these matrix models via a least-squares approach at a global scale.

These techniques are well established and effective, however, as these are statistical approaches, they cannot provide the identity of individual atoms. This concept can be illustrated by considering a repeated "heads/tails" fair coin toss, where we only know the final sum of heads/tails outcomes, but cannot observe each toss. Whilst we can identify that in a large series of coin tosses, the outcome will be $50 \%$ heads $/ 50 \%$ tails, we cannot determine what the result of a specific coin toss (e.g. the first) was, as we did not directly observe it. This information is simply unavailable.

In the context of APT, this means that, although we can measure composition, we cannot directly utilise any methods that require the querying of individual atomic identities within the $3 \mathrm{D}$ volume, such as voxelisation, proxigrams or even simple composition analyses in cases involving overlaps. Nevertheless, the problem is partly solvable. Returning to the coin toss analogy, whilst it is not possible to be certain about each toss, we can have reasonable confidence about the statistics of a large subset of throws, almost regardless of how we decide to construct such a subset (every 2nd throw, the first 20 throws, the last 20 throws, etc).

Such an approach is detailed here, where we generate a pseudo-dataset where peak decomposition has been applied at a local scale. This is shown in Fig. 6, where two concentrations of atoms are shown, and an overlap exists for a single peak. The coloured atoms can be unambiguously identified, however the grey atoms cannot. However, examining a small volume surrounding each we can perform a localised decomposition. By examining a single atom on the left and on the right's vicinity, we observe two differing spectra, and thus two widely different solutions to the decomposition. We can use the decomposition to assign the atom based upon its locally identified probability of belonging to the unresolved overlap, and reassign randomly.

As an example, we examine a scenario whereby a ${ }^{12} \mathrm{C}_{2}{ }^{+}$and ${ }^{48} \mathrm{Ti}^{2+}$ overlap occurs at mass $24.00 / 23.974$, in a high-Mn steel containing a TiC particle, shown in Fig. 7. Here, a model is given to utilise the ratio of the primary (24 peak) and secondary counts (23, $23.5,24.5,25$ peaks) within local volumes defined by a sphere centred around each unidentified ion. The sphere radius is specified by the user, to estimate the overlap probability at each point as per a standard decomposition. The selection of radius depends upon several factors, such as the level of spatial fidelity required, and the minimum number of atoms to be able to resolve the overlap - these parameters are best chosen with a-priori knowledge of the materials science problem to be solved and the dataset to which the algorithm is to be applied.

Using the locally decomposed relative compositions of the two identities (Ti and $\mathrm{C}$ ), the ion is randomly assigned a new chemical

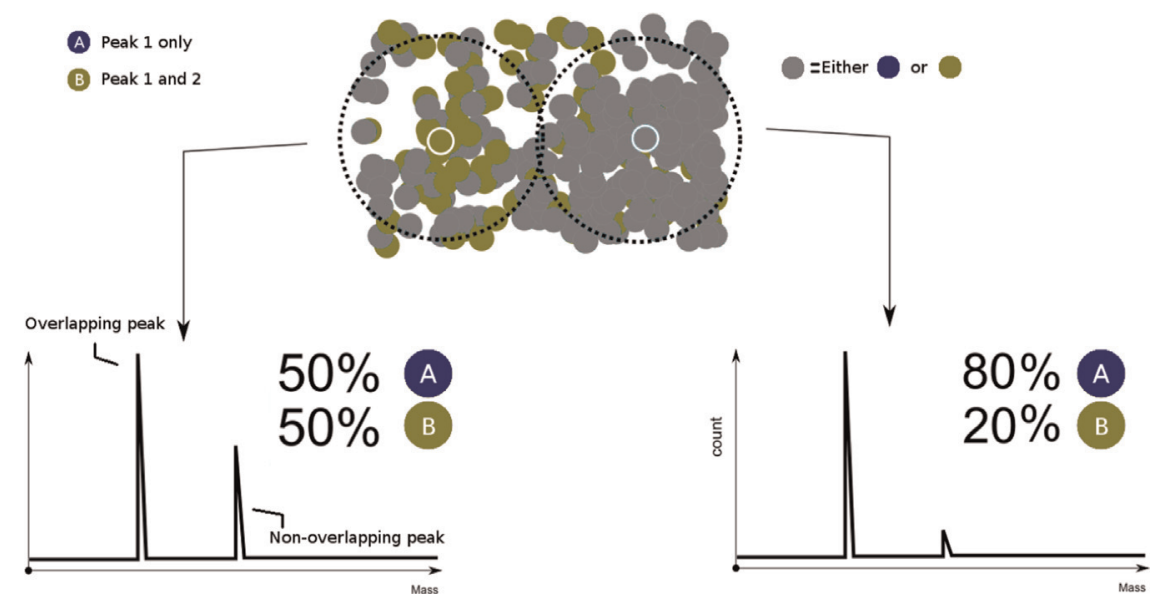

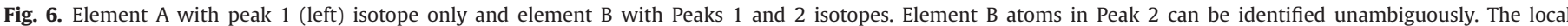

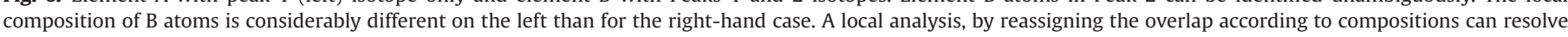

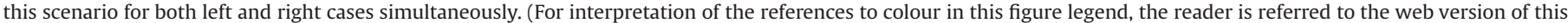
article.) 

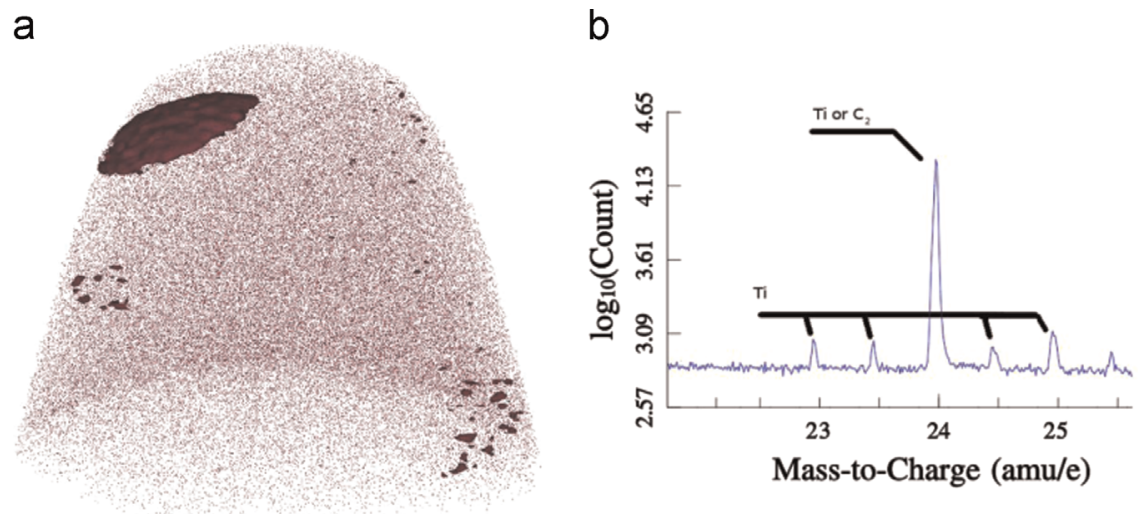

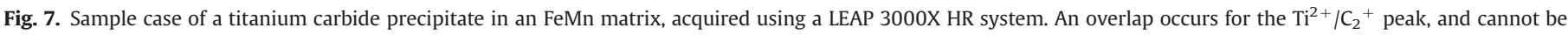
unambiguously assigned to Ti or $\mathrm{C}_{2}$.

identity by this probabilistic weight. This preserves the local composition and also generates a highly probable point distribution, with no overlap ambiguity. The only parameters are the length scale used for the sphere radius when generating local spectra, and the species involved in the overlaps. These parameters control a trade-off between using a sufficiently large radius to have sufficient statistics to be able to stably estimate composition and having a small enough radius to provide spatial resolution.

The utility of this method lies in its ability to enable standard composition estimation tools to be applied unmodified. Due to lack of bias in the random reassignment, the expected values of any composition measurement (i.e. the average composition within any volume) are corrected. This is an advantage over any bias in a single-valued assignment (e.g. assigning a spectral peak as either Ti or C specifically), as is typically performed.

As shown for the TiC particle case above, the Ti/C overlap in the uncorrected dataset prevents the correct stoichiometry from being estimated by either a proxigram or composition profile as shown in Fig. 8. Using the corrected dataset $(r=1 \mathrm{~nm})$, we can now use the same proxigram tool to observe the corrected composition for the TiC particle, as estimated by this method. Compared to the uncorrected version, the ratio moves far closer to the expected Ti50-C50 stoichiometry. Some carbon loss is observed, but this is a common problem in APT [121-125].

The primary drawback to this method is that it mixes data from the sampling volume of each ion, i.e. the volume from which the composition was drawn. In the implementation used here, the sampling volume is a sphere, centred around each ion, resulting in a sampling blur equivalent to the sphere used as the selection volume. Practically speaking, the blur will widen step profiles, such as in proxigrams and composition profiles. Fig. 9 shows the blurring of a simulation containing an initially sharp interface, where the algorithm has sampled data from both sides of the interface. In the pure regions away from the interface, there is no incorrect reassignment, as the composition goes to $100 \%$, and thus no discernable blurring occurs. However as can be seen from the profile across the interface, the blurring has converted the initial step into a ramp, equal to 2 distance units wide.

The statistical certainties for any specific generated dataset are naturally finite, as the method in turns depends upon the level of error in the decomposition process itself. A pragmatic solution to obtaining confidence in the analysis could be to generate multiple "corrected" datasets in order to estimate the confidence levels to which measurements on the corrected dataset can be reported.

Whilst parameter selection remains a concern, the method provides a useful option for the minimisation of spatial overlap concerns in arbitrary algorithms, to provide improved estimation of spatial variations in composition.

\section{Data dimensionality}

Atom probe tomography is usually characterised as a three dimensional chemical imaging tool. However, the challenges in the interpretation of data reconstruction are complex and influenced by numerous complex and interacting parameters [126-128] (Fig. 10). At this stage of development in the field, we do not have the full equivalent in atom probe tomography of a contrast transfer function as we have in transmission electron microscopy $[129,130]$.

When such explicit functional relationships based on theory and/or phenomenological data are not available to capture all the diversity of variables (Fig. 10), one needs to explore ways to statistically capture such information. As discussed earlier in this paper, there are a multitude of different types of information that can be empirically identified, measured or some in cases even modelled. The challenge is to find a way to simultaneously obtain the cross-correlations among all these parametric studies. Hence we can describe atom probe data as having a "high
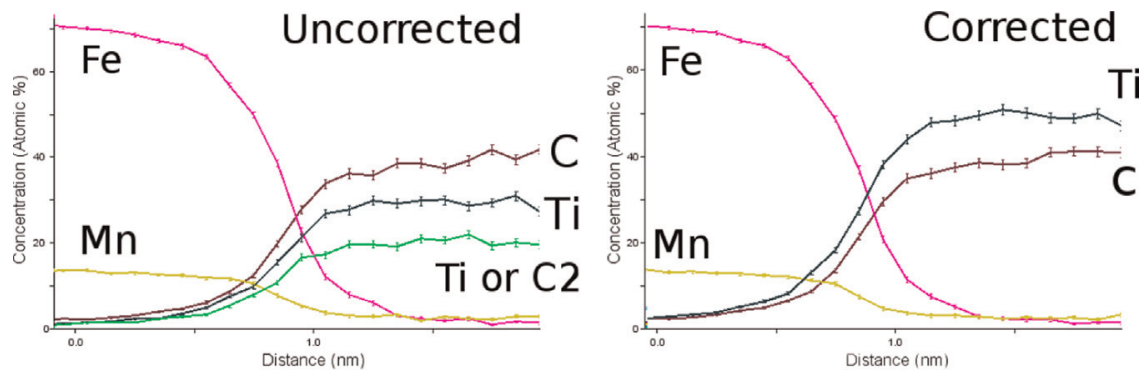

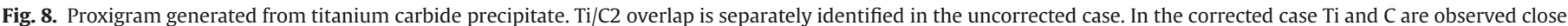
to their expected stoichiometery. 

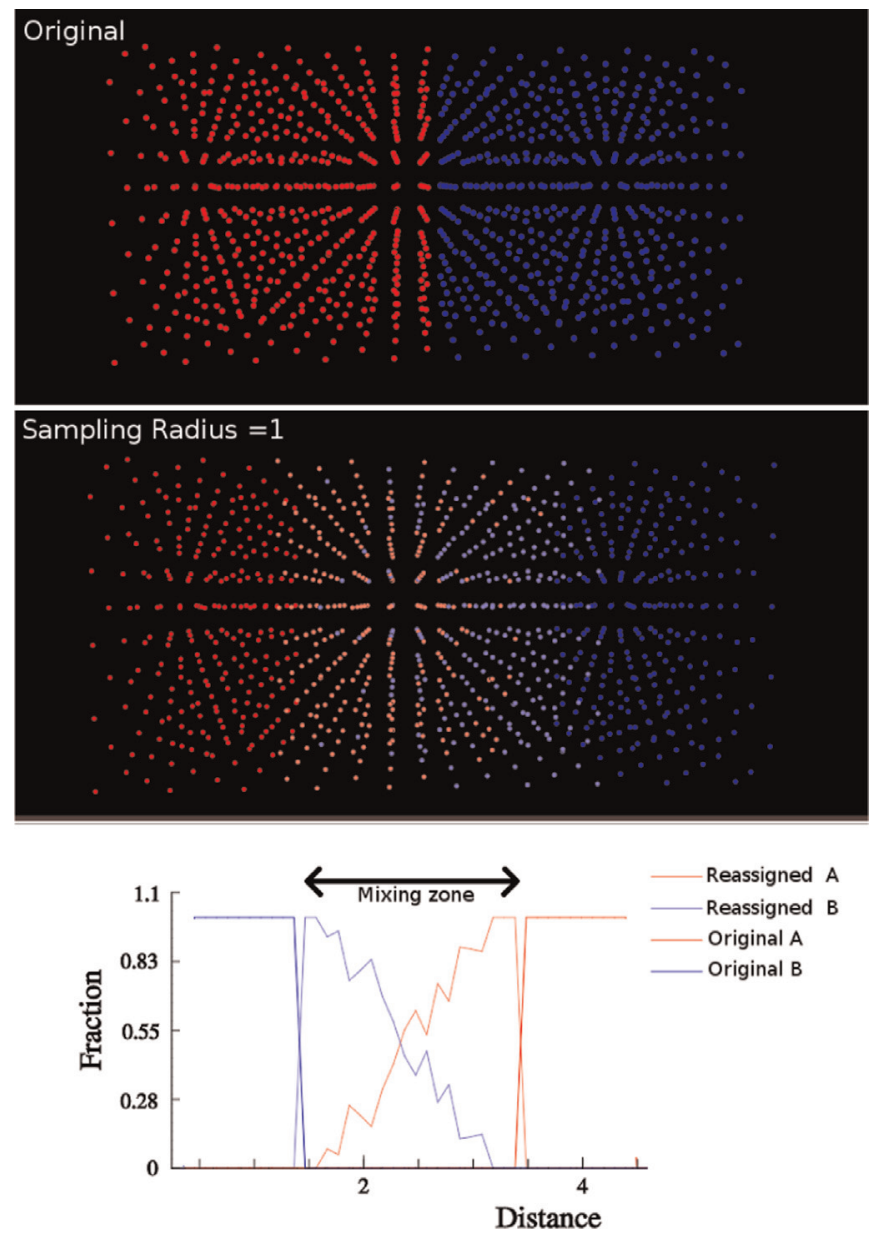

Fig. 9. Simulation demonstrating blurring of sharp interfaces, an artefact of the deconvolution approach suggested here. In this simulation A and B atoms are randomly reassigned according to their local compositions using a sphere of radius $1 \mathrm{~nm}$. The sharp interface is now a mixture of A and B, transitioning from one to the other.

dimensionality" since for each of the millions of atoms detected there are multiple parameters associated with each hit including spatial coordinates, instrumental parameters, composition, the evaporation physics and parameters associated with the reconstruction analysis.

A key step is to find ways to map the correlations between all these parameters (and a diverse array of other attributes that underlie them), in a manner that can be interpreted. Hence there is a need to map or "project" this high dimensional information into lower dimensions, preferably two or three dimensions, which is the only way these correlations can be visualised. In this "reduced" dimensional space, we can then seek to find patterns and associations of data that permit us to unravel the complexity and truly "mine" the rich information embedded in atom probe images and spectra. The process of reducing the dimensionality of data mathematically is one that has to be done carefully, to avoid losing or distorting true correlations between characteristics in the original data set $[73,131-134]$. There are numerous techniques to accomplish this and in the following discussion we shall provide a couple of brief examples of the value of using such methods.

When accounting for the multivariate nature of most materials chemistry problems and the numerous variables and parameters that can be associated with instrument operation and data acquisition, this data cube is actually an $n$-dimensional hypercube. Hence the challenge is to "unfold" the high dimensional data matrix and identify the key or principal characteristics that capture the key spatial relationships of chemistry in a chemical image.

\subsection{Enhancing peak discrimination in atom probe mass spectra}

Much as Fourier transformations or derivative analyses are used to amplify signals in techniques such as secondary ion mass spectrometry (SIMS), it is possible to mathematically enhance the features in mass spectra using an eigenvalue decomposition. The typical APT output is a single mass spectrum. This approach converts this spectrum into multiple spectra capturing various representations of information. Therefore instead of calibrating isotope identity by decomposing a single spectrum, Broderick et al. [134] applied data dimensionality reduction methods to decompose multiple spectra capturing "hidden" APT information. This process is schematically shown in Fig. 11. Similar approaches have also been developed by others [116,135].

Fig. 12 shows the contribution of individual isotopes to the entire time-of-flight (TOF) spectra. Although the TOF spectrum displays only two peaks, the spectrum has been decomposed into four components based on enhancing the signal through eigenvalue decomposition, and calibrating the signal based on six unique spectra instead of a single spectrum. The output from APT is the dotted black line, while the output from this analysis is the four solid coloured lines. Therefore, despite isotopic overlap in the mass spectra, this approach defines which isotope is associated with each collected ion.

\subsection{Refining spatial distribution maps (SDMs)}

Previously mentioned in Section 3 in relation to atom probe crystallography, SDMs build a picture of the average atomic neighbourhoods in the dataset by directly examining nearestneighbour inter-atomic distances along a particular crystallographic direction (Fig. 13). An SDM is a record of all interatomic position vectors in a dataset from zero to some pre-described vector length. SDMs can be computed and the results can be displayed in multiple crystallographic directions, measuring

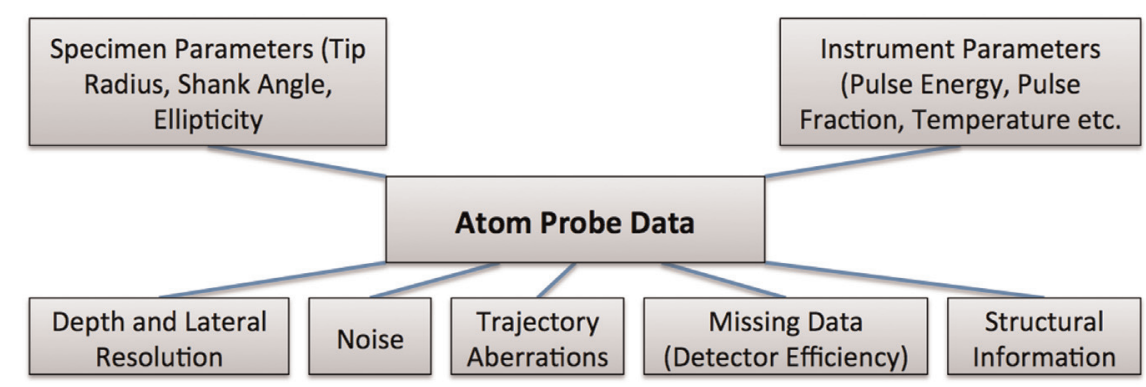

Fig. 10. Multi-dimensional parametric influences on the quantitative interpretation of atom probe data. 


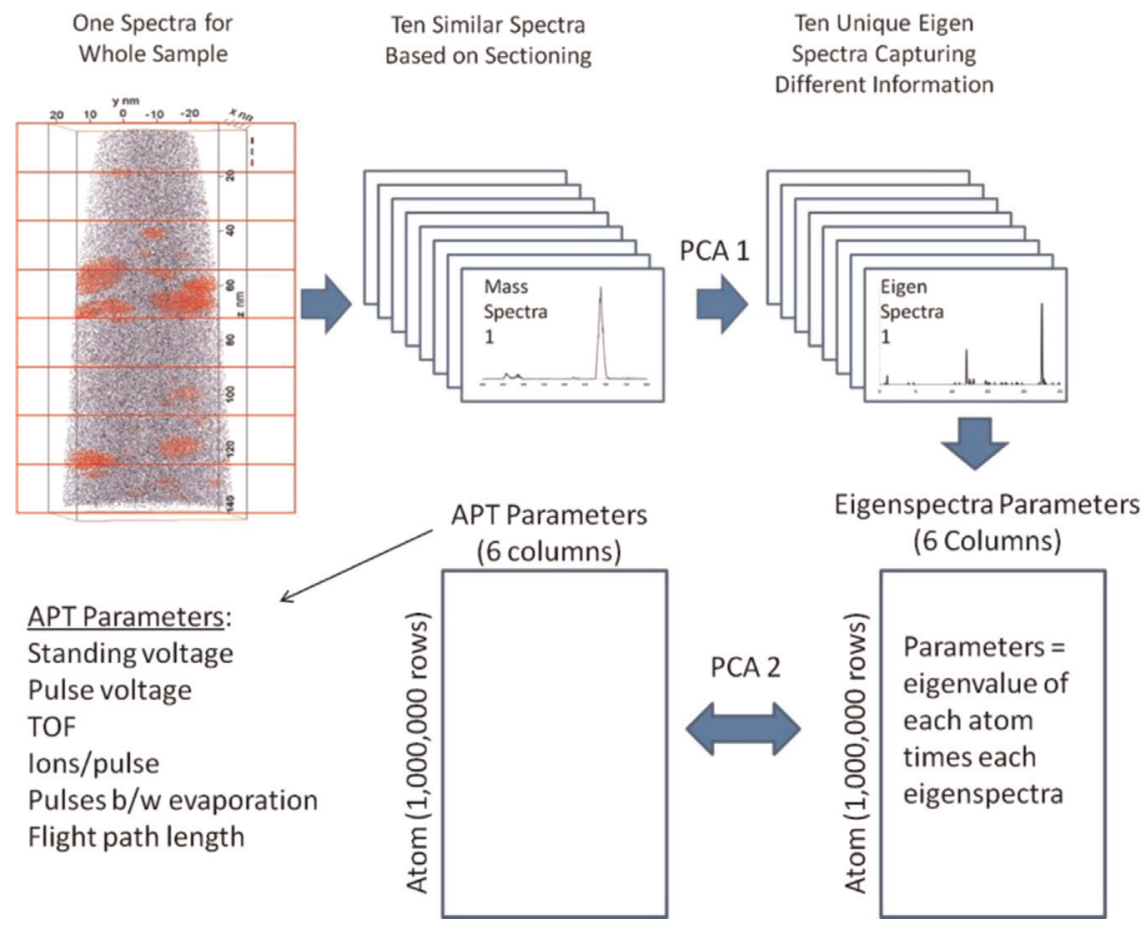

Fig. 11. Schematic of eigenvalue deconvolution approach to capture the influence of instrumental parameters on the mass spectrum [134].

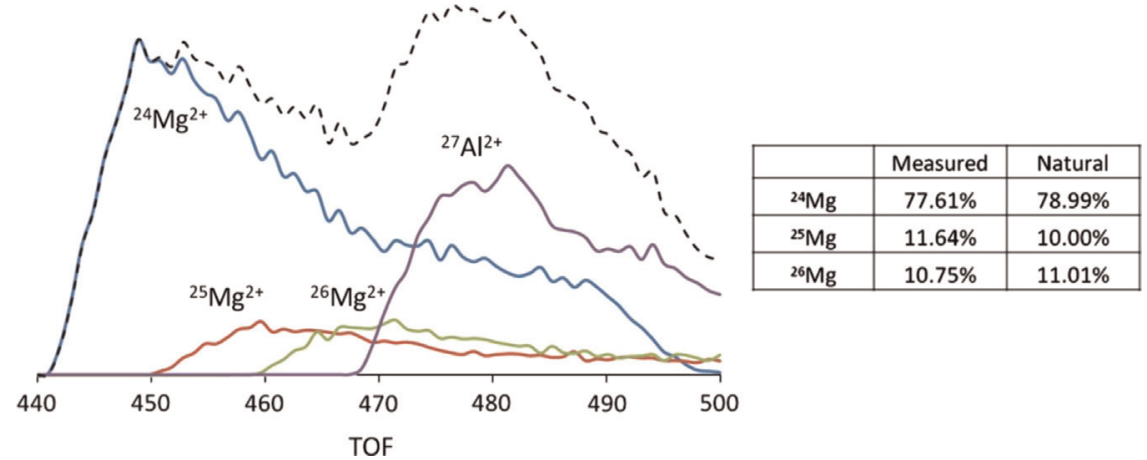

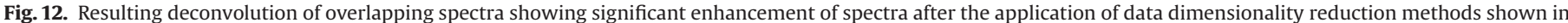
Fig. 11 [134]. (For interpretation of the references to colour in this figure legend, the reader is referred to the web version of this article.)

a

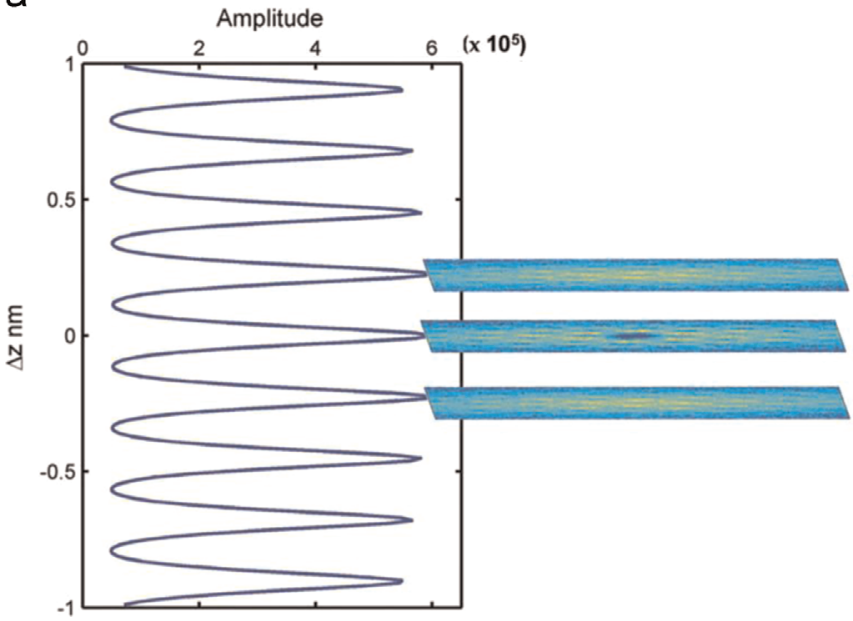

b

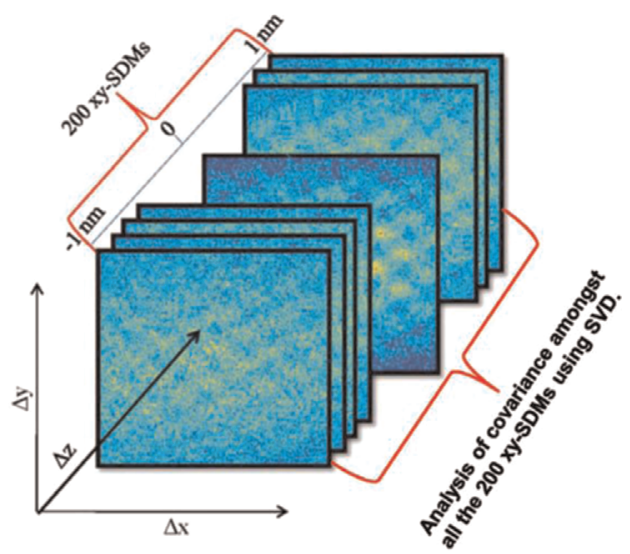

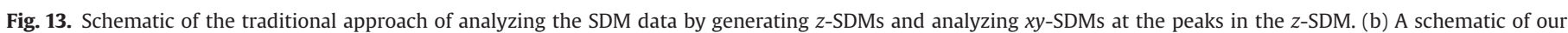
approach wherein the structural information embedded in the SDMs is extracted by analyzing the covariance among all the $x y$-SDMs using SVD (from [136]). 


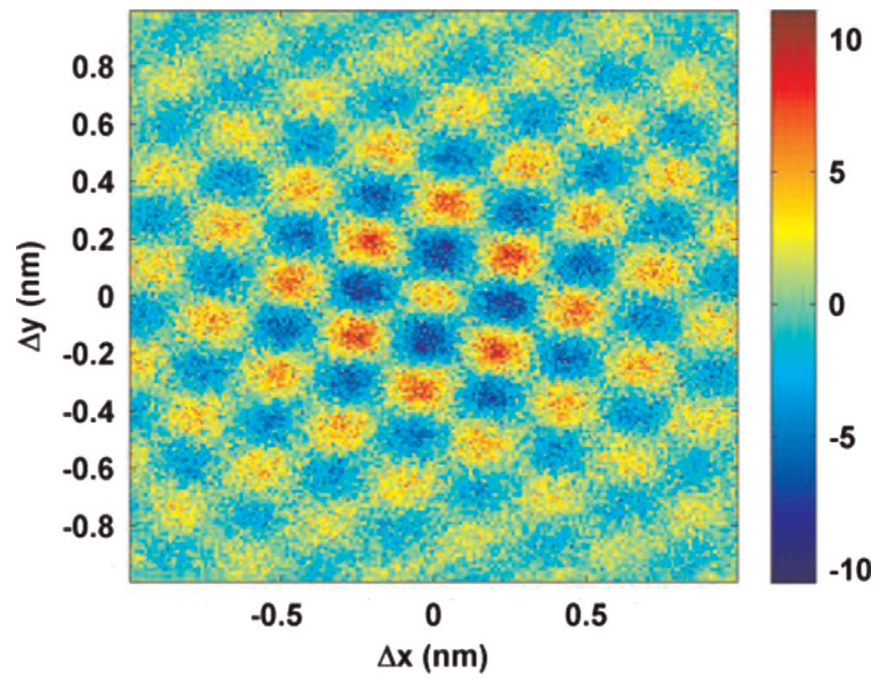

Fig. 14. The enhancement in the signal-to-noise ratio in the refined $x y$-SDMs [136].

interplanar spacings and crystallographic angles, and effectively provide a form of radial distribution function. They can be used for finding crystal lattices, correcting lattice distortions in reconstructed images, quantifying trajectory aberrations, quantifying spatial resolution, quantifying chemical ordering, determining orientation relationships, extracting radial distribution functions, and measuring ion detection efficiency. However the presence of missing data and spatial uncertainty poses a big challenge and one needs to account for ways to capture the intrinsic high dimensionality of an SDM. Suram and Rajan [73] for example have applied data dimensionality reduction methods such as singular value decomposition to capture not only the position of atomic planes but also the structural covariance among the neighbourhood planes. This permits one to classify the SDM data into noise, structurally relevant information and aberrations (Fig. 14).

\section{Summary and conclusion}

With recent advances in instrumentation $[137,138]$ and specimen preparation techniques [139-141] allowing atom probe to be applied to almost any solid material site, quantitative analysis of atom probe data remains as one of the most important areas of development in atom probe microscopy. This paper has provided a survey of some of the approaches through which one can extract and significantly enhance the interpretation of atom probe data. They range from the use of data mining and pattern recognition methods to the application of statistical learning methods. Improvements in instrumentation may provide further capabilities for improving spatial and mass resolution, which may reduce or eliminate the need for some of the approaches described here. Nevertheless, data analysis and data mining techniques can still provide insights into chemistry and structure of datasets, beyond what may be traditionally considered to be the information content of a given system. The role of mining information from atom probe data is not only a critical step in the analysis of information coming from experiments, but also provides a basis of quantitatively linking the interpretation of data to atomistic modelling and simulations of atom probe tomography. This is a rich area for future research.

Further advances in methods to mine atom probe data, and their integration into available analysis programs will undoubtedly aid complete extraction of the available experimental information. Collectively when these methods are harnessed in the analysis of atom probe experiments, it is possible to dramatically improve the scope of this powerful technique.

\section{Acknowledgement}

The authors acknowledge the facilities and the scientific and technical assistance of the Australian Microscopy \& Microanalysis Research Facility at the Australian Centre for Microscopy \& Microanalysis at the University of Sydney. BG acknowledges that he is a full time employee of Elsevier Ltd. but declares no conflict of interest as his contribution to this article corresponds to his activity out of office hours. KR acknowledges support from the AFOSR grants FA9550-11-1-0158 and FA9550-12-1-0456; Wilkinson Professorship of Interdisciplinary Engineering at Iowa State University.

\section{References}

[1] B. Gault, M.P. Moody, J.M. Cairney, S.P. Ringer, Atom Probe Microscopy, Springer, 2012

[2] M.K. Miller, Atom Probe Tomography: Analysis at the Atomic Level, 2000.

[3] T.F. Kelly, M.K. Miller, Invited review article: atom probe tomography, Rev. Sci. Instrum. 78 (2007) 031101-031120 031101

[4] D.J. Larson, T.J. Prosa, R.M. Ulfig, B.P. Geiser, T.F. Kelly, Atom Probe Tomography: A Users Guide, Springer, 2013.

[5] M.K. Miller, F. R.G. Forbes, Atom Probe Tomography, Spinger, 2014.

[6] A.V. Ceguerra, A.J. Breen, L.T. Stephenson, P.J. Felfer, V.J. Araullo-Peters, P. V. Liddicoat, X. Cui, L. Yao, D. Haley, M.P. Moody, B. Gault, J.M. Cairney, S. P. Ringer, The rise of computational techniques in atom probe microscopy, Curr. Opin. Solid State Mater. Sci. 17 (2013) 224-235.

[7] O.C. Hellman, J.B. du Rivage, D.N. Seidman, Efficient sampling for three-dimensional atom probe microscopy data, Ultramicroscopy 95 (2003) 199-205.

[8] O.C. Hellman, J.A. Vandenbroucke, J. Rusing, D. Isheim, D.N. Seidman, Analysis of three-dimensional atom-probe data by the proximity histogram, MIcrosc. Microanal. 6 (2000) 437-444.

[9] E.A. Marquis, J.M. Hyde, Applications of atom-probe tomography to the characterisation of solute behaviours, Mater. Sci. Eng. R 69 (2010) 37-62.

[10] Andrew J. Breen, Michael P. Moody, Anna V. Ceguerra, Baptiste Gault, Vicente J. Araullo-Peters, Simon P. Ringer., Restoring the lattice of Si-based atom probe reconstructions for enhanced information on dopant positioning. Ultramicroscopy 2015, (in press), this issue.

[11] L. Yao, B. Gault, J.M. Cairney, S.P. Ringer, On the multiplicity of field evaporation events in atom probe: a new dimension to the analysis of mass spectra, Philos. Mag. Lett. 90 (2010) 121-129.

[12] L. Yao, J.M. Cairney, B. Gault, C. Zhu, S.P. Ringer, Correlating spatial, tempora and chemical information in atom probe data: new insights from multiple evaporation in microalloyed steels, Philos. Mag. Lett. 93 (2013) 299-306.

[13] D.W. Saxey, Correlated ion analysis and the interpretation of atom probe mass spectra, Ultramicroscopy 111 (2011) 473-479.

[14] E.A. Marquis, J.M. Hyde, Applications of atom-probe tomography to the characterisation of solute behaviours, Mater. Sci. Eng. R: Rep. 69 (2010) 37-62.

[15] R.K.W. Marceau, A. De Vaucorbeil, G. Sha, S.P. Ringer, W.J. Poole, Analysis of strengthening in AA6111 during the early stages of aging: atom probe tomography and yield stress modelling. Acta Mater. 61 (2013) 7285-7303.

[16] R.K.W. Marceau, G. Sha, R. Ferragut, A. Dupasquier, S.P. Ringer, Solute clustering in $\mathrm{Al}-\mathrm{Cu}-\mathrm{Mg}$ alloys during the early stages of elevated temperature ageing, Acta Mater. 58 (2010) 4923-4939.

[17] T. Alam, M. Chaturvedi, S.P. Ringer, J. Cairney, The early stages of ageing in the Ni-based superalloy, inconel 718, Mater. Forum 34 (2008) 1-5.

[18] T. Alam, M. Chaturvedi, S.P. Ringer, J.M. Cairney, Precipitation and clustering in the early stages of ageing in Inconel 718, Mater. Sci. Eng. A 527 (2010) $7770-7774$.

[19] K.Y. Xie, T. Zheng, J.M. Cairney, H. Kaul, J.G. Williams, F.J. Barbaro, C. R. Killmore, S.P. Ringer, Strengthening from Nb-rich clusters in a Nb-microalloyed steel, Scr. Mater. 66 (2012) 710-713.

[20] P.D. Styman, J.M. Hyde, K. Wilford, A. Morley, G.D.W. Smith, Precipitation in long term thermally aged high copper, high nickel model RPV steel welds, Prog. Nucl. Energy 57 (2012) 86-92.

[21] M. Bachhav, G. Robert Odette, E.A. Marquis, $\alpha^{\prime}$ Precipitation in neutron-irradiated Fe-Cr alloys, Scr. Mater. 74 (2014) 48-51.

[22] Y. Chen, P.H. Chou, E.A. Marquis, Quantitative atom probe tomography characterization of microstructures in a proton irradiated 304 stainless steel J. Nucl. Mater. 451 (2014) 130-136.

[23] E.A. Marquis, Atomic-scale characterization of nuclear reactor materials, Trans. Am. Nucl. Soc. (2010) 744. 
[24] H. Gnaser, S. Gutsch, M. Wahl, R. Schiller, M. Kopnarski, D. Hiller M. Zacharias., Phosphorus doping of Si nanocrystals embedded in silicon oxynitride determined by atom probe tomography, J. Appl. Phys. 115 (2014) 034304.

[25] L. Li, Y. Guo, X.Y. Cui, R. Zheng, K. Ohtani, C. Kong, A.V. Ceguerra, M.P. Moody, J.D. Ye, H.H. Tan, C. Jagadish, H. Liu, C. Stampfl, H. Ohno, S.P. Ringer, F. Matsukura, Magnetism of Co-doped $\mathrm{ZnO}$ epitaxially grown on a $\mathrm{ZnO}$ substrate, Phys. Rev. B - Condens. Matter Mater. Phys. 85 (2012).

[26] W.K. Yeoh, B. Gault, X. Cui, C. Zhu, M.P. Moody, Direct observation of local potassium variation and its correlation to electronic inhomogeneity in $\left(\mathrm{Ba}_{1-x} \mathrm{~K}_{x}\right) \mathrm{Fe}_{2} \mathrm{As}_{2}$ pnictide, Phys. Rev. Lett. 106 (2011) 247002.

[27] T.E. Kelly, D.J. Larson, K. Thompson, R.L. Alvis, J.H. Bunton, J.D. Olson, B. R. Gorman, Atom probe tomography of electronic materials, Annu. Rev. Mater. Res. (2007) 681-727.

[28] T.J. Godfrey, M.G. Hetherington, J.M. Sassen, G.D.W. Smith, The characterization of spinodal structures in Duplex CF3 steels, J. Phys. 49 (1988) 421-426.

[29] M.K. Miller, A. Cerezo, M.G. Hetherington, G.D.W. Smith, Atom Probe Field Ion Microscopy, Oxford Science Publications - Clarendon Press, 1996.

[30] M.P. Moody, L.T. Stephenson, A.V. Ceguerra, S.P. Ringer, Quantitative binomial distribution analyses of nanoscale like-solute atom clustering and segregation in atom probe tomography data, Microsc. Res. Tech. 71 (2008) $542-550$.

[31] E. Camus, C. Abromeit, Analysis of conventional and 3-dimensional atomprobe data for multiphase materials, J. Appl. Phys. 75 (1994) 2373-2382.

[32] A. Shariq, T. Al-Kassab, R. Kirchheim, R.B. Schwarz, Exploring the next neighbourhood relationship in amorphous alloys utilizing atom probe tomography, Ultramicroscopy 107 (2007) 773-780.

[33] T. Philippe, F. De Geuser, S. Duguay, W. Lefebvre, O. Cojocaru-Miredin, G. Da Costa, D. Blavette, Clustering and nearest neighbour distances in atom-probe tomography, Ultramicroscopy 109 (2009) 1304-1309.

[34] L.T. Stephenson, M.P. Moody, P.V. Liddicoat, S.P. Ringer, New techniques for the analysis of fine-scaled clustering phenomena within atom probe tomography (APT) data, MIcrosc. Microanal. 13 (2007) 448-463.

[35] F.D. Geuser, W. Lefebvre, Determination of matrix composition based on solute-solute nearest-neighbor distances in atom probe tomography, Microsc. Res. Tech. 74 (2011) 257-263.

[36] M. Wada, On the thermally activated field evaporation of surface atoms, Surf Sci. 145 (1984) 451-465.

[37] B. Gault, F. Danoix, K. Hoummada, D. Mangelinck, L. H., Impact of directional walk on atom probe microanalysis, Ultramicroscopy 113 (2012).

[38] J.M. Cowley, An approximate theory of order in alloys, Phys. Rev. Lett. 77 (1950) 669-675

[39] D. de Fontaine, The number of independent pair correlation functions in multicomponent systems, J. Appl. Crystallogr. 4 (1971) 15.

[40] A. Ceguerra, R.C. Powles, M.P. Moody, S.P. Ringer, Quantitative description of atomic architecture in solid solutions: a generalized theory for multicomponent short-range order, Phys. Rev. B 82 (2010) 132201.

[41] A.V. Ceguerra, M.P. Moody, R.C. Powles, T.C. Petersen, R.K.W. Marceau, S.P. Ringer, Short-range order in multicomponent materials, Acta Crystallogr. A 68 (2012) 547-560.

[42] C.K. Sudbrack, R.D. Noebe, D.N. Seidman, Direct observations of nucleation in a nondilute multicomponent alloy, Phys. Rev. B - Condens. Matter Mater. Phys. 73 (2006) 2212101.

[43] F. De Geuser, W. Lefebvre, D. Blavette, 3D atom probe study of solute atoms clustering during natural ageing and pre-ageing of an $\mathrm{Al}-\mathrm{Mg}-\mathrm{Si}$ alloy, Philos. Mag. Lett. 86 (2006) 227-234.

[44] A. Shariq, T. Al-Kassab, R. Kirchheim, Studying nearest neighbor correlations by atom probe tomography (APT) in metallic glasses as exemplified for Fe40Ni40B20 glassy ribbons, J. Alloy. Compd. 512 (2012) 270-277.

[45] D. Haley, T. Petersen, G. Barton, S.P. Ringer, Influence of field evaporation on radial distribution functions in atom probe tomography, Philos. Mag. 89 (2009) 925-943

[46] C.A. Johnson, J.H. Klotz, The atom probe and Markov chain statistics of clustering, Technometrics 16 (1974) 483-493.

[47] M.K. Miller, Decomposition of bulk metallic glasses, Mater. Sci. Eng. A 250 (1998) 133-140.

[48] M.K. Miller, D.J. Larson, R.B. Schwarz, Y. He, Decomposition in Pd40Ni40P20 metallic glass, Mater. Sci. Eng. A 250 (1998) 141-145.

[49] M.K. Miller, T.D. Shen, R.B. Schwarz, Atom probe tomography study of the decomposition of a bulk metallic glass, Intermetallics 10 (2002) 1047-1052.

[50] B. Gault, X.Y. Cui, M.P. Moody, F. De Geuser, S.C. Du, S.P. Ringer, Atom probe microscopy investigation of Mg site occupancy within delta 'precipitates' in an Al-Mg-Li alloy, Scr. Mater. 66 (2012) 903-906.

[51] T. Rademacher, T. Al-Kassab, J. Deges, R. Kirchheim, Ordering and site occupancy of D03 ordered $\mathrm{Fe} 3 \mathrm{Al}-5$ at\% $\mathrm{Cr}$ evaluated by means of atom probe tomography, Ultramicroscopy 111 (2011) 719-724.

[52] J.A. Horton, M.K. Miller, Atom probe analysis of grain boundaries in rapidlysolidified Ni3Al, Acta Metall. 35 (1987) 133-141.

[53] K. Hono, A. Chiba, T. Sakurai, S. Hanada, Determination of site occupation probability of $\mathrm{Cu}$ in Ni3Al by atom-probe field ion microscopy, Acta Metall. Mater. 40 (1992) 419-425.

[54] T. Boll, T. Al-Kassab, Y. Yuan, Z.G. Liu, Investigation of the site occupation of atoms in pure and doped TiAl/Ti3 Al intermetallic, Ultramicroscopy 107 (2007) 796-801.

[55] T. Al-Kassab, Y. Yuan, C. Kluthe, T. Boll, Z.G. Liu, Investigation of the ordering and atomic site occupancies of Nb-doped TiAl/Ti3Al intermetallics, Surf. Interface Anal. 39 (2007) 257-261.

[56] S. Kim, G.D.W. Smith, AP-FIM investigation on $\gamma$-based titanium aluminides, Mater. Sci. Eng. A 239-240 (1997) 229-234.

[57] A. Almazouzi, H. Numakura, M. Koiwa, K. Hono, T. Sakurai, Site occupation preference of fe in Ni3Al: an atom-probe study, Intermetallics 5 (1997) 37-43.

[58] E.A. Marquis, D.N. Seidman, A subnanoscale study of $\mathrm{Mg}$ segregation at $\mathrm{Al} /$ Al3Sc interfaces, Microsc. Microanal. 8 (2002) 1100-1101.

[59] B.P. Geiser, T.F. Kelly, D.J. Larson, J. Schneir, J.P. Roberts, Spatial distribution maps for atom probe tomography, Microsc. Microanal. 13 (2007) 437-447.

[60] J. Li, X. Gu, T.C. Hufnagel, Using fluctuation microscopy to characterize structural order in metallic glasses, Microsc. Microanal. 9 (2003) 509-515.

[61] H.W. Sheng, W.K. Luo, F.M. Alamgir, J.M. Bai, E. Ma, Atomic packing and short-to-medium range order in metallic glasses, Nature 439 (2006) 419-425.

[62] R.K.W. Marceau, A. Ceguerra, A.J. Breen, D. Raabe, S.P. Ringer, Quantitative chemical-structure evaluation using atom probe tomography: short-range order analysis of Fe-Al, Ultramicroscopy 157 (2015) 12-20.

[63] M.P. Moody, B. Gault, L. Stephenson, R.K.W. Marceau, P. R.C., A. Ceguerra, A. J. Breen, S.P. Ringer, Lattice rectification in atom probe tomography: toward true three-dimensional atomic microscopy, Microsc. Microanal. 17 (2011) 226-239.

[64] Y.C. Chen, D.N. Seidman, On the atomic resolution of a field ion microscope, Surf. Sci. 26 (1971) 61-84.

[65] E.W. Müller, Resolution of the atomic structure of a metal surface by the field ion microscope, J. Appl. Phys. 27 (1956) 474.

[66] V. Araullo-Peters, B. Gault, F.D. Geuser, A. Deschamps, J.M. Cairney, Microstructural evolution during ageing of $\mathrm{Al}-\mathrm{Cu}-\mathrm{Li}-\mathrm{X}$ alloys, Acta Mater. 66 (2014) 199-208.

[67] V. Araullo-Peters, A.J. Breen, A. Ceguerra, B. Gault, S.P. Ringer, J.M. Cairney, A new systematic framework for crystallographic analysis of atom probe data, Ultramicroscopy 154 (2015) 7-14.

[68] M.P. Moody, B. Gault, L.T. Stephenson, D. Haley, S.P. Ringer, Qualification of the tomographic reconstruction in atom probe by advanced spatial distribution map techniques, Ultramicroscopy 109 (2009) 815-824.

[69] F. Vurpillot, G. Da Costa, A. Menand, D. Blavette, Structural analyses in threedimensional atom probe: a Fourier transform approach, J. Microsc. - Oxf. 203 (2001) 295-302.

[70] P.J. Warren, A. Cerezo, G.D.W. Smith, Observation of atomic planes in 3DAP analysis, Ultramicroscopy 73 (1998) 261-266.

[71] F. Vurpillot, F. De Geuser, G. Da Costa, D. Blavette, Application of Fourier transform and autocorrelation to cluster identification in the three-dimensional atom probe4, J. Microsc. 216 (2004) 234-240.

[72] L. Yao, M.P. Moody, J.M. Cairney, A.V. Ceguerra, C. Zhu, D. Haley, S.P. Ringer, Determining crystal structure with atom probe tomography via 3D Hough transformations, Ultramicroscopy 111 (2011) 458-463.

[73] S.K. Suram., K. Rajan, Refining spatial distribution maps for atom probe tomography via data dimensionality reduction methods, J. Microsc. Microanal. 18 (2012) 941-952.

[74] B. Gault, F. de Geuser, L.T. Stephenson, M.P. Moody, B.C. Muddle, S.P. Ringer, Estimation of the reconstruction parameters for atom probe tomography, Microsc. Microanal. 14 (2008) 296-305.

[75] D.J. Larson, B. Gault, B.P. Geiser, F. Vurpillot, Atom probe tomography spatial reconstruction: status and directions, Curr. Opin. Solid State Mater. Sci. 17 (2013) 236-247.

[76] P.V. Liddicoat, X.Z. Liao, Y.H. Zhao, Y.T. Zhu, M.Y. Murashkin, E.J. Lavernia, R. Z. Valiev, S.P. Ringer, Nanostructural hierarchy increases the strength of aluminium alloys, Nat. Commun. 1 (2010).

[77] V.J. Araullo-Peters, B. Gault, S.L. Shrestha, L. Yao, M.P. Moody, S.P. Ringer, J. M. Cairney, Atom probe crystallography: atomic-scale 3D orientation mapping, Scr. Mater. 66 (2012) 907-910.

[78] B. Gault, M.P. Moody, F. de Geuser, G. Tsafnat, A. La Fontaine, L.T. Stephenson, D. Haley, S.P. Ringer, Advances in the calibration of atom probe tomographic reconstruction, J. Appl. Phys. 105 (2009).

[79] L. Yao, S.P. Ringer, J.M. Cairney, M.K. Miller, The anatomy of grain boundaries: their structure and atomic level solute distribution, Scr. Mater. 69 (2013) 622-625.

[80] V. Araullo-Peters, B. Gault, F. De Geuser, A. Deschamps, C. Sigli, J.M. Cairney, Microstructural evolution during ageing of AA2198: an atom probe study, Acta Mater. 66 (2014) 199-208.

[81] D. Blavette, P. Duval, L. Letellier, M. Guttmann, TEM atomic-scale investigation of grain boundary microchemistry in astroloy nickel base superalloys, Acta Mater. 44 (1996) 4995-5005.

[82] B. Gault, M.P. Moody, J.M. Cairney, S.P. Ringer, Atom probe crystallography, Mater. Today 15 (2012) 378-386.

[83] L. Karlsson, H.O. Andren, H. Norden, Grain boundary segregation in an austenitic stainless-steel containing boron - an atom probe study, Scr. Metall. 16 (1982) 297-302.

[84] B.W. Krakauer, D.N. Seidman, Absolute atomic-scale measurements of the Gibbsian interfacial excess of solute at internal interfaces, Phys. Rev. B 48 (1993) 6724-6727.

[85] M.K. Miller, G.D.W. Smith, Atom-probe analysis of interfacial segregation, Appl. Surf. Sci. 87-8 (1995) 243-250.

[86] H. Norden, H.O. Andren, Atom-probe analysis of grain-boundary segregation, Surf. Interface Anal. 12 (1988) 179-184. 
[87] K. Seto, D.J. Larson, P.J. Warren, G.D.W. Smith, Grain boundary segregation in boron added interstitial free steels studied by 3-dimensional atom probe, Scr. Mater. 40 (1999) 1029-1034.

[88] A.R. Waugh, M.J. Southon, Surface-analysis and grain-boundary segregation measurements using atom-probe techniques, Surf. Sci. 89 (1979) 718-724.

[89] J. Weidow, H.-O. Andren, Grain and phase boundary segregation in WC-Co with small V, Cr or Mn additions, Acta Mater. 58 (2010) 3888-3894.

[90] D.J. Larson, Atom probe characterization of nanomagnetic materials, Thin Solid Films 505 (2006) 16-21.

[91] D.J. Larson, A.K. Petford-Long, Y.Q. Ma, A. Cerezo, Information storage materials: nanoscale characterisation by three-dimensional atom probe analysis, Acta Mater. 52 (2004) 2847-2862.

[92] M.P. Moody, F. Tang, B. Gault, S.P. Ringer, J.M. Cairney, Atom probe crystallography: characterization of grain boundary orientation relationships in nanocrystalline aluminium, Ultramicroscopy 111 (2011) 493-499.

[93] M. Ngamo, S. Duguay, P. Pichler, K. Daoud, P. Pareige, Characterization of arsenic segregation at $\mathrm{Si} / \mathrm{SiO}_{2}$ interface by $3 \mathrm{D}$ atom probe tomography, Thin Solid Films 518 (2010) 2402-2405.

[94] R. Rachbauer, S. Massl, E. Stergar, P. Felfer, P.H. Mayrhofer, Atom probe specimen preparation and 3D interfacial study of Ti-Al-N thin films, Surf. Coat. Technol. 204 (2010) 1811-1816.

[95] P.J. Felfer, B. Gault, G. Sha, L.T. Stephenson, S.P. Ringer, J.M. Cairney, A new approach to the determination of concentration profiles in atom probe tomography, Microsc. Microanal. 18 (2015) 359-364.

[96] M.P.A. Fisher, M. Wortis, Curvature corrections to the surface tension of fluid drops: Landau theory and a scaling hypothesis, Phys. Rev. B 29 (1984) 6252-6260.

[97] K.E. Yoon, R.D. Noebe, O.C. Hellman, D.N. Seidman, Dependence of interfacial excess on the threshold value of the isoconcentration surface, Surf. Interface Anal. 36 (2004) 594-597.

[98] J.W. Gibbs, The Collected Works of J. Willard Gibbs, Longmans, Green and Co., New York, 1902.

[99] W.E. Lorensen, H.E. Cline, Marching cubes: a high resolution 3D surface construction algorithm, SIGGRAPH, Comput. Graph. 21 (1987) 163-169.

[100] P. Felfer, A. Ceguerra, S. Ringer, J. Cairney, Detecting and extracting clusters in atom probe data: a simple,automated method using Voronoi cells, Ultramicroscopy 150 (2015) 30-36.

[101] P.W. Trimby, Y. Cao, Z. Chen, S. Han, K.J. Hemker, J. Lian, X. Liao, P. Rottmann, S. Samudrala, J. Sun, J.T. Wang, J. Wheeler, J.M. Cairney, Characterizing deformed ultrafine-grained and nanocrystalline materials using transmission Kikuchi diffraction in a scanning electron microscope, Acta Mater. 62 (2013) 69-80.

[102] M.K. Miller, L. Yao, Limits of detectability for clusters and solute segregation to grain boundaries, Curr. Opin. Solid State Mater. Sci. 17 (2013) 203-210.

[103] P. Felfer, P. Benndorf, A. Masters, T. Maschmeyer, J.M. Cairney, Revealing the distribution of the atoms within individual bimetallic catalyst nanoparticles, Angew. Chem. 126 (2014) 11372-11375.

[104] P. Felfer, A. Ceguerra, S. Ringer, J. Cairney, Applying computational geometry techniques for advanced feature analysis in atom probe data, Ultramicroscopy 132 (2013) 100-106.

[105] S.K. Samudrala, P.J. Felfer, V.J. Araullo-Peters, Y. Cao, X.Z. Liao, J.M. Cairney, Atom probe for the study of segregation in nanocrystalline materials, Ultramicroscopy 132 (2013) 158-163.

[106] F. Tang, T. Alam, M.P. Moody, B. Gault, J.M. Cairney, Challenges associated with the characterisation of nanocrystalline materials using atom probe tomography, , 654-656 (1-3) (2010) 2366-2369, http://dx.doi.org/10.4028/ www.scientific.net/MSF.654-656.2366.

[107] P.J. Felfer, B. Scherrer, J. Demeulemeister, W. Vandervorst, J.M. Cairney, Mapping interfacial excess in atom probe data, Ultramicroscopy, this issue.

[108] T.F. Kelly, M.K. Miller, Invited review article: atom probe tomography, Rev. Sci. Instrum. 78 (2007).

[109] E.A. Guggenheim, N.K. Adam, The thermodynamics of adsorption at the surface of solutions, Proc. R. Soc. Lond. Ser. A: Containing Pap. Math. Phys. Charact. 139 (1993) 218-236.

[110] D.N. Seidman, B.W. Krakauer, D. Udler, Atomic scale studies of solute-atom segregation at grain boundaries: experiments and simulations, J. Phys. Chem. Solids 55 (1994) 1035-1057.

[111] F. Aurenhammer, Voronoi diagrams-a survey of a fundamental geometric data structure, ACM Comput. Surv. 23 (1991) 345-405.

[112] L. Yao, S.P. Ringer, J.M. Cairney, M.K. Miller, The anatomy of grain boundaries: their structure and atomic-level solute distribution, Scr. Mater. 69 (2013) 622-625.

[113] B. Mamyrin, V. Karataev, D. Shmikk, V. Zagulin, The mass reflectron, a new non-magnetic time-of-flight mass spectrometer with high resolution, Sov. J. Exp. Theor. Phys. 64 (1973) 82-89.

[114] S. Sijbrandij, A. Cerezo, T. Godfrey, G. Smith, Improvements in the mass re solution of the three-dimensional atom probe, Appl. Surf. Sci. 94 (1996) 428-433.

[115] T.E. Kelly, Kinetic-energy discrimination for atom probe tomography, Microsc. Microanal. 17 (2011) 1-14.
[116] M.R. Keenan, V.S. Smentkowski, R.M. Ulfig, E. Oltman, D.J. Larson, T.F. Kelly, Atomic-scale phase composition through multivariate statistical analysis of atom probe tomography data, Microsc. Microanal. 17 (2011) 418-430.

[117] M. Murayama, K. Hono, H. Hirukawa, T. Ohmura, S. Matsuoka, The combined effect of molybdenum and nitrogen on the fatigued microstructure of 316 type austenitic stainless steel, Scr. Mater. 41 (1999) 467-473.

[118] J. Angseryd, F. Liu, H.-O. Andrén, S. Gerstl, M. Thuvander, Quantitative APT analysis of Ti(C,N), Ultramicroscopy 111 (2011) 609-614.

[119] M. Sonestedt, K. Stiller, Using atom probe tomography to analyse MAX-phase materials, Ultramicroscopy (2011) 642-647.

[120] L. Johnson, M. Thuvander, K. Stiller, M. Odén, L. Hultman, Blind deconvolution of time-of-flight mass spectra from atom probe tomography, Ultramicroscopy 132 (2013) 60-64.

[121] H.S. Kitaguchi, S. Lozano-Perez, M.P. Moody, Quantitative analysis of carbon in cementite using pulsed laser atom probe, Ultramicroscopy 147 (2014) 51

[122] R.K.W. Marceau, P. Choi, D. Raabe, Understanding the detection of carbon in austenitic high-Mn steel using atom probe tomography, Ultramicroscopy 132 (2013) 239-247.

[123] Y. Kobayashi, J. Takahashi, K. Kawakami, Anomalous distribution in atom map of solute carbon in steel, Ultramicroscopy 111 (2011) 600-603.

[124] W. Sha, L. Chang, G.D.W. Smith, C. Liu, E.J. Mittemeijer, Some aspects of atomprobe analysis of FeC and FeN systems, Surf. Sci. 266 (1992) 416-423.

[125] M.W. Thuvander, J.; Angseryd, J. Falk, L.K.L. Liu, F.L. Sonestedt, M. Stiller, K. H.-O. Andrén, Quantitative atom probe analysis of carbides, Ultramicroscopy (2011) 604-608.

[126] B. Gault, S.T. Loi, V. Araullo-Peters, L.T. Stephenson, M.P. Moody, S. Shrethsa R.K.W. Marceau, L. Yao, J.M. Cairney, S.P. Ringer, Dynamic reconstruction for atom probe tomography, Ultramicroscopy 111 (2011) 1619-1624.

[127] B. Gault, M.P. Moody, E.A. Marquis, F.D. Geuser, B.P. Geiser, D.J. Larson, T, F. Kelly, S.P. Ringer, G.D.W. Smith, Tomographic reconstruction in atom probe microscopy: past, present...future? Microsc. Microanal. 15 (2009) 10-11.

[128] D.J. Larson, B. Gault, B.P. Geiser, F. De Geuser, F. Vurpillot, Atom probe tomography spatial reconstruction: status and directions, Curr. Opin. Solid State Mater. Sci. 17 (2013) 236-247.

[129] S. J.C.H., Experimental High-Resolution Electron Microscopy, 2nd ed., Oxford University Press, New York, 1988

[130] E.J. Kirkland, Advanced Computing in Electron Microscopy, Plenum Press, New York, 1998.

[131] A. Bryden, S. Broderick, S.K. Suram, K. Kaluskar, R. LeSar, K. Rajan, Interactive visualization of atom probe tomography data at full fidelity, Ultramicroscopy 132 (2013) 129-135.

[132] J. Peralta, S. Broderick, K. Rajan, Mapping energetics of atom probe evaporation events through first principles calculations, Ultramicroscopy 132 (2013) 143-151.

[133] S. Samudrala, O. Wodo, S.K. Suram, S. Broderick, K. Rajan, B. Ganapathysubramanian, A graph-theoretic approach for characterization of precipitates in alloys from atom probe tomography data, Comput. Mater Sci. 77 (2013) 335-342.

[134] S.R. Broderick, A. Bryden, S.K. Suram, K. Rajan, Data mining for isotope discrimination in atom probe tomography, Ultramicroscopy 132 (2013) $121-128$

[135] F. Tang, T. Alam, M.P. Moody, B. Gault, J.M. Cairney, Challenges associated with the characterisation of nanocrystalline materials using atom probe tomography, Mater. Sci. Forum 654-656 (1-3) (2010) 2366-2369.

[136] S.K. Suram, K. Rajan, Calibration of reconstruction parameters in atom probe tomography using a single crystallographic orientation, Ultramicroscopy 132 (2013) 136-142.

[137] B. Gault, F. Vurpillot, A. Vella, M. Gilbert, A. Menand, D. Blavette, B. Deconihout, Design of a femtosecond laser assisted tomographic atom probe, Rev. Sci. Instrum. 77 (2006).

[138] J.H. Bunton, J.D. Olson, D.R. Lenz, T.E. Kelly, Advances in pulsed-laser atom probe: instrument and specimen design for optimum performance, Microsc. Microanal. 13 (2007) 418-427.

[139] K. Thompson, D. Lawrence, D.J. Larson, J.D. Olson, T.F. Kelly, B. Gorman, In situ site-specific specimen preparation for atom probe tomography, Ultramicroscopy 107 (2007) 131-139.

[140] M.K. Miller, K.F. Russell, K. Thompson, R. Alvis, D.J. Larson, Review of atom probe FIB-based specimen preparation methods, Microsc. Microanal. 13 (2007) 428-436.

[141] P.J. Felfer, T. Alam, S.P. Ringer, J.M. Cairney, A reproducible method for damage-free site-specific preparation of atom probe tips from interfaces, Microsc. Res. Tech. 75 (2012) 484-491.

[142] Atomically resolved tomography to directly inform simulations for structureproperty relationships. Michael P. Moody, Anna V. Ceguerra, Andrew J. Breen, Xiang Yuan Cui, Baptiste Gault, Leigh T. Stephenson, Ross K. W. Marceau, Rebecca C. Powles, Simon P. Ringer, Nat. Commun. 5, Article number: 5501 http://dx.doi.org/10.1038/ncomms6501. 